\title{
Counting the Number of $\tau$-Exceptional Sequences over Nakayama Algebras
}

\author{
Dixy Msapato ${ }^{1}[$
}

Received: 5 April 2020 / Accepted: 28 April 2021 / Published online: 2 June 2021

(C) The Author(s) 2021

\begin{abstract}
The notion of a $\tau$-exceptional sequence was introduced by Buan and Marsh in (2018) as a generalisation of an exceptional sequence for finite dimensional algebras. We calculate the number of complete $\tau$-exceptional sequences over certain classes of Nakayama algebras. In some cases, we obtain closed formulas which also count other well known combinatorial objects, and exceptional sequences of path algebras of Dynkin quivers.
\end{abstract}

Keywords $\tau$-Exceptional sequence Exceptional sequence $\cdot$ Nakayama algebras · $\tau$-Perpendicular category $\cdot$ Restricted Fubini numbers

Mathematics Subject Classification (2020) 16G10 - 16G20 (Primary), 05A10 - 05A15 . 05A18 $\cdot$ 05A19 (Secondary)

\section{Introduction}

Let $A$ be a finite dimensional algebra over a field $\mathbb{F}$, where $\mathbb{F}$ is algebraically closed. Let $\bmod A$ be the category of finitely generated left $A$-modules. A left $A$-module $M$ is called exceptional if $\operatorname{Hom}(M, M) \cong \mathbb{F}$ and $\operatorname{Ext}_{A}^{i}(M, M)=0$ for $i \geq 1$. A sequence of indecomposable modules $\left(M_{1}, M_{2}, \ldots, M_{r}\right)$ is called an exceptional sequence if for each pair $\left(M_{l}, M_{j}\right)$ with $1 \leq l<j \leq r$, we have that $\operatorname{Hom}\left(M_{j}, M_{l}\right)=\operatorname{Ext}_{A}^{i}\left(M_{j}, M_{l}\right)=0$ for $i \geq 1$, and each $M_{k}$ is exceptional for $1 \leq k \leq r$. Exceptional sequences were first introduced in the context of algebraic geometry by [6], [15] and [14].

Exceptional sequences exhibit some interesting behaviours. It was shown by CrawleyBoevey [10] and Ringel [25] that there is a transitive braid group action on the set of

This research was supported by an EPSRC Doctoral Training Partnership (reference EP/R513258/1) through the University of Leeds. The author also wishes to thank their supervisor, Bethany Marsh for their support. Lastly, but not least, the author also thanks the referees of this paper for their comments on an earlier version of the paper.

Presented by: Henning Krause

Dixy Msapato

mmdmm@leeds.ac.uk 
exceptional sequences. Igusa and Schiffler give [16] a characterisation of exceptional sequences for hereditary algebras using the fact that the product of the corresponding reflections is the inverse Coxeter element of the Weyl group. The exceptional sequences for $\bmod \mathbb{A}_{r}$, where $\mathbb{A}_{r}$ is the path algebra of a Dynkin type A quiver are classified in [12] using combinatorial objects called strand diagrams. The exceptional sequences over path algebras of type A, were also characterised using non-crossing spanning trees in [3]. A natural question for exceptional sequences is to ask how many there are. The number of them has been computed for all the hereditary Dynkin algebras in [28] and [22].

Exceptional sequences have been subject to a number of generalisations. Igusa and Todorov introduced the signed exceptional sequences in [17]. More recently, weak exceptional sequences were introduced and studied by Sen in [30]. Finally, Buan and Marsh introduced in [7] the signed $\tau$-exceptional sequences and $\tau$-exceptional sequences. It is $\tau$ exceptional sequences which are the subject of this paper. An $A$-module $M$ is called $\tau$-rigid if $\operatorname{Hom}(M, \tau M)=0$, see Definition 0.1 in [2]. The $\tau$-perpendicular category of $M$ in $\bmod A$ is the subcategory $J(M)=M^{\perp} \cap^{\perp}(\tau M)$, see Definition 3.3 in [18]. A sequence of indecomposable modules $\left(M_{1}, M_{2}, \ldots, M_{r}\right)$ in $\bmod A$ is called a $\tau$-exceptional sequence if $M_{r}$ is $\tau$-rigid in $\bmod A$ and $\left(M_{1}, M_{2}, \ldots, M_{r-1}\right)$ is a $\tau$-exceptional sequence in $J\left(M_{r}\right)$.

Our main results are derivations of closed formulas for the number of complete $\tau$ exceptional sequences in the module categories of certain Nakayama algebras. Most notably, we see that the complete $\tau$-exceptional sequences over the linear radical square zero Nakayama algebras $\Gamma_{n}^{2}$ are counted by the restricted Fubini numbers $F_{n, \leqslant 2}$ [21]. The numbers $F_{n, \leqslant 2}$ count the number of ordered set partitions of the set $\{1,2, \ldots, n\}$ with blocks of size at most two. In the case for the cyclic Nakayama algebra $\Lambda_{n}^{n}$, we get that the complete $\tau$-exceptional sequences are counted by the sequence $n^{n}$. We remark that this sequence also counts the number of complete exceptional sequences for the hereditary Dykin algebras of quivers of type B and C, as shown in [22], and full weak exceptional sequences over $\Lambda_{n}^{n}$, see [29, Theorem 3.5]. In fact, we show that the complete $\tau$-exceptional sequences over $\Lambda_{n}^{n}$ coincide with the full weak exceptional sequences over $\Lambda_{n}^{n}$, see Corollary 6.13 .

We remark that Buan and Marsh showed in [7] that there is a bijection between complete signed $\tau$-exceptional sequences and basic ordered support $\tau$-tilting modules over a finite dimensional algebra. So, one way of counting signed $\tau$-exceptional sequences would be to count ordered support $\tau$-tilting modules, but this would not give the number of (unsigned) $\tau$-exceptional sequences, which is what we consider here. In this direction, Asai [4] gave a recurrence relation for the number of support $\tau$-tilting modules over Nakayama algebras with a linearly oriented type A quiver. Adachi [1] also gave a recurrence relation for the number of $\tau$-tilting modules over the same algebras as Asai. More recently Gao and Schiffler [11] have extended the recurrence relations of Adachi and Asai to $\tau$-tilting modules and support $\tau$-tilting modules over Nakayama algebras whose quiver is an oriented cycle. In a paper of Sen [29], the number of exceptional sequences over the linear radical square zero Nakayama algebras $\Gamma_{n}^{2}$ are counted. However, to date the number of exceptional sequences for other classes of Nakayama algebras have not been counted.

This paper is organised as follows: In Section 2 we fix some notation and recall definitions. In Section 3, we state and prove preliminary results which we use in the latter sections to prove our main results of the paper. Our main results of this section state that under certain assumptions, the $\tau$-exceptional sequences of $\bmod A$ are obtained by interleaving $\tau$-exceptional sequences of certain subcategories of mod $A$. In Section 4 , we count the number of complete $\tau$-exceptional sequences for the linear radical square zero Nakayama 
algebras $\Gamma_{n}^{2}$. We derive a recurrence and closed formula for the number of complete $\tau$ exceptional sequences in this case. In Section 5, we deal with the case of cyclic radical square zero Nakayama algebras $\Lambda_{n}^{2}$. We derive a closed formula for the number of complete $\tau$-exceptional sequences in this case. We also derive a formula for the number of complete $\tau$-exceptional sequences of $\Lambda_{n}^{2}$ in terms of the number of complete $\tau$-exceptional sequences of $\Gamma_{n}^{2}$. In Section 6, we count the number of complete $\tau$-exceptional sequences over the cyclic Nakayama algebras $\Lambda_{n}^{n}$. We derive a recurrence and closed formula for the number of complete $\tau$-exceptional sequences in this case. Section 7 deals with the linear Nakayama algebras $\Gamma_{n}^{n-1}$. We derive a recurrence relation for the number of complete $\tau$-exceptional sequences in this case, and show that the corresponding exponential generating function satisfies a certain first order linear ordinary differential equation involving Lambert's W function. Section 8 concludes the paper by giving a justification of why we only consider the above the classes of Nakayama algebras.

\section{Definitions and Notation}

Let $A$ be a basic finite dimensional algebra over a field $\mathbb{F}$ which is algebraically closed. Let $\bmod A$ be the category of finite dimensional left $A$-modules. Denote by $\mathcal{P}(A)$ the full subcategory of projective objects in $\bmod A$. If $\mathcal{T}$ is a subcategory of $\bmod A$, we say an $A$ module $M$ in $\mathcal{T}$ is Ext-projective in $\mathcal{T}$ if $\operatorname{Ext}_{A}^{1}(M, \mathcal{T})=0$; that is to say $\operatorname{Ext}_{A}^{1}(M, T)=0$ for all $T \in \mathcal{T}$. We will then write $\mathcal{P}(\mathcal{T})$ to denote the direct sum of the indecomposable Ext-projective modules in $\mathcal{T}$. In everything that follows, we make the assumption that subcategories are full, and closed under isomorphism. We will also take all objects to be basic where possible, and they will be considered up to isomorphism.

For an additive category $\mathcal{C}$, and an object $X$ in $\mathcal{C}$, we denote by add $X$ the additive subcategory of $\mathcal{C}$ generated by $X$. This is the subcategory of $\mathcal{C}$ with objects the direct summands of direct sums of copies of $X$. For a subcategory $\mathcal{X} \subseteq \mathcal{C}$, we define ${ }^{\perp} \mathcal{X}:=\{Y \in$ $\mathcal{C}: \operatorname{Hom}(Y, X)=0$ for all $X \in \mathcal{X}\}$ and we similarly define $\mathcal{X}^{\perp}$. If $\mathcal{C}$ is skeletally small and Krull-Schmidt, we denote by ind $(\mathcal{C})$ the set of isomorphism classes of indecomposable objects in $\mathcal{C}$. For any basic object $X$ in $\mathcal{C}$, let $\delta(X)$ denote the number of indecomposable direct summands of $X$. We fix $\delta(A)$ to be $n$, where $n \geq 1$ is a positive integer.

Let $\tau$ denote the Auslander-Reiten translate of $\bmod A$.

Definition 2.1 $\tau$-rigid and $\tau$-tilting [2, Definition 0.1]. A left $A$-module $M$ is said to be $\tau$-rigid if $\operatorname{Hom}(M, \tau M)=0$. If furthermore $\delta(M)=n$, we say that $M$ is $\tau$-tilting.

Definition 2.2 $\tau$-perpendicular category [18, Definition 3.3]. Let $M$ be a basic $\tau$-rigid left $A$-module. The $\tau$-perpendicular category associated to $M$ is the subcategory of $\bmod A$ given by $J_{\bmod A}(M)$ : $=M^{\perp} \cap^{\perp}(\tau M)$. If there is no risk of ambiguity, we will write $J(M)$ for the subcategory $J_{\bmod A}(M)$.

Definition 2.3 $\tau$-exceptional sequence [7, Definition 1.3]. Let $k$ be a positive integer. A sequence of indecomposable modules $\left(M_{1}, M_{2}, \ldots, M_{k}\right)$ in mod $A$ is called a $\tau$-exceptional sequence in $\bmod A$ if $M_{k}$ is $\tau$-rigid in $\bmod A$ and $\left(M_{1}, M_{2}, \ldots, M_{k-1}\right)$ is a $\tau$-exceptional sequence in $J\left(M_{k}\right)$. If $k=n$ we say that the sequence is a complete $\tau$-exceptional sequence.

Let $Q$ be a finite quiver on $n$ vertices labelled by the set $\{1,2, \ldots, n\}$. A path $p$ in $Q$ from the vertex $v_{1}$ to the vertex $v_{m}$ is a sequence of vertices $p=\left(v_{1}, v_{2}, v_{3}, \ldots, v_{m-1}, v_{m}\right)$ 
such that $\left(v_{j}, v_{j+1}\right)$ is an arrow in $Q$ for all $1 \leq j \leq m-1$. The positive integer $m$ is called the length of $p$ and it is denoted by $l(p)$. The path algebra $\mathbb{F} Q$ of the quiver $Q$ is the $\mathbb{F}$-algebra with basis all paths of $Q$, and multiplication is defined by concatenation of paths. The the arrow ideal $R_{Q}$ of $\mathbb{F} Q$ is defined to be the two-sided ideal generated by all arrows in $Q$. The arrow ideal has a vector space decomposition given by,

$$
R_{Q}=\mathbb{F} Q_{1} \oplus \mathbb{F} Q_{2} \oplus \cdots \oplus \mathbb{F} Q_{l} \oplus \ldots
$$

where $\mathbb{F} Q_{l}$ is the subspace of $\mathbb{F} Q$ with basis the set $Q_{l}$ of paths of length $l$. The $l^{\text {th }}$ power of the arrow ideal, denoted by $R_{Q}^{l}$ is given by,

$$
R_{Q}^{l}=\bigoplus_{m \geq l} \mathbb{F} Q_{m},
$$

it has a basis consisting of all paths of length greater than or equal to $l$.

For a positive integer $n \geq 1$, let $A_{n}$ denote the linearly oriented quiver with $n$ vertices,

$$
1 \stackrel{\alpha_{1}}{\longrightarrow} 2 \stackrel{\alpha_{2}}{\longrightarrow} 3 \stackrel{\alpha_{3}}{\longrightarrow} \ldots \stackrel{\alpha_{n-2}}{\longrightarrow} n-1 \stackrel{\alpha_{n-1}}{\longrightarrow} n \text {. }
$$

Let $C_{n}$ be the linearly oriented $n$-cycle.

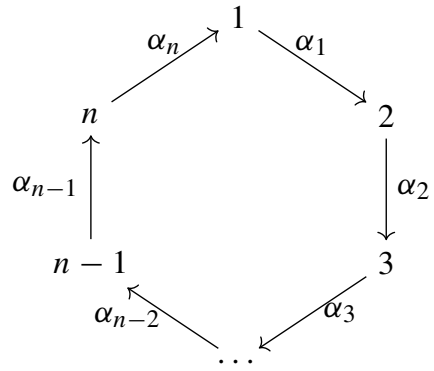

We denote by $\Gamma_{n}^{t}$ the Nakayama algebra $\mathbb{F} A_{n} / R_{Q}^{t}$ and by $\Lambda_{n}^{t}$ the self injective Nakayama algebra $\mathbb{F} C_{n} / R_{Q}^{t}$ where $2 \leq t \leq n$. Throughout the text, we will write $P_{i}$ for the indecomposable projective module at vertex $i$ of the underlying quiver of the algebra $A$ in question. Likewise we will write $S_{i}$ for the simple $A$-module at vertex $i$.

Definition 2.4 [27, Definition 3.1]. Let $Q$ be a finite quiver.

1. Two paths $p=\left(v_{1}, v_{2}, \ldots, v_{m}\right)$ and $p^{\prime}=\left(v_{1}^{\prime}, v_{2}^{\prime}, \ldots, v_{m^{\prime}}^{\prime}\right)$ in $Q$ are called parallel if $v_{1}=v_{1}^{\prime}$ and $v_{m}=v_{m^{\prime}}^{\prime}$.

2. A relation $\rho$ in $Q$ is an $\mathbb{F}$-linear combination $\rho=\sum_{c} \lambda_{c} c$ of parallel paths with $l(c) \geq$ 2 , and $\lambda_{c} \in \mathbb{F}$.

For a positive integer $n \geq 1$, we will write $(a)_{n}$ to stand for $a$ modulo $n$. We will also write $[i, j]_{n}$ for the set $\left\{(i)_{n},(i+1)_{n}, \ldots,(j-1)_{n},(j)_{n}\right\}$.

\section{Preliminary Results}

In this section, we will state and prove results which will be used in later sections to calculate the number of $\tau$-exceptional sequences over the algebras $\Gamma_{n}^{t}$ and $\Lambda_{n}^{t}$. However, our main results are much more general and they apply to other finite dimensional algebras. For this section, we fix an arbitrary finite dimensional $\mathbb{F}$-algebra $A$. 
Proposition 3.1 [2, Theorem 2.10]. Let $M$ be a $\tau$-rigid A-module. Then the following holds:

1. The module $M$ is Ext-projective in ${ }^{\perp}(\tau M)$, which is to say that $M$ is in add $\left(\mathcal{P}\left({ }^{\perp}(\tau M)\right)\right)$.

2. The module $T_{M}:=\mathcal{P}\left({ }^{\perp}(\tau M)\right)$ is a $\tau$-tilting A-module.

The $A$-module $T_{M}$ is called the Bongartz completion of $M$ in $\bmod A$.

Example 3.2 Let $A$ be the algebra $\Gamma_{3}^{2}$ given by the quiver

$$
1 \stackrel{\alpha_{1}}{\longrightarrow} 2 \stackrel{\alpha_{2}}{\longrightarrow} 3,
$$

subject to the relation $\alpha \beta=0$. The Auslander-Reiten quiver of $\bmod \Gamma_{3}^{2}$ is as follows,

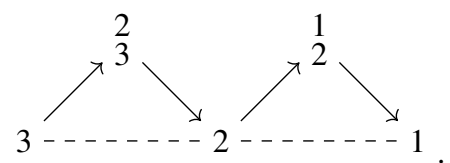

For the $\Gamma_{3}^{2}$-module $M=1$, ind $\left({ }^{\perp}(\tau 1)\right)=\operatorname{ind}\left({ }^{\perp} 2\right)=\left\{3,{ }_{2}^{1}, 1\right\}$. Therefore it is easy to see that $T_{1}=\mathcal{P}\left({ }^{\perp}(\tau 1)\right)=3 \oplus{ }_{2}^{1} \oplus 1$. It is also easy to observe that $T_{1}$ is indeed a $\tau$-tilting $\Gamma_{3}^{2}$-module.

Proposition 3.3 [2, Lemma 2.1]. Let I be an ideal of $A$, and let $M, N$ be $A / I$-modules. Then we have the following:

1. If $\operatorname{Hom}_{A}(M, \tau N)=0$ then $\operatorname{Hom}_{A / I}\left(M, \tau_{A / I} N\right)=0$.

2. If $I=\langle e\rangle$ for some idempotent $e \in A$, then it is the case that $\operatorname{Hom}_{A}(M, \tau N)=0$ if and only if $\operatorname{Hom}_{A / I}\left(M, \tau_{A / I} N\right)=0$.

The following lemma is well known and it will be important in this paper.

Lemma 3.4 Let $Q$ be a finite simple quiver with vertex set $\{1,2, \ldots, n\}$. Let I be the ideal of $\mathbb{F} Q$ generated by relations on $Q$ where each relation is a path in $Q$ and take $A=\mathbb{F} Q / I$. For some $j \in\{1,2, \ldots, n\}$ let $Q^{(j)}$ be the quiver obtained from $Q$ by removing the vertex $j$ and any arrows incident to $j$. Let $I^{(j)} \subset I$ be the ideal of $\mathbb{F} Q$ generated by the generating relations of $I$ defined by paths of $Q$ not containing the vertex $j$ and take $B=\mathbb{F} Q^{(j)} / I^{j}$. Then $B \cong A /\left\langle e_{j}\right\rangle$ as an $\mathbb{F}$-algebra, where $e_{j}$ is the idempotent at vertex $j$ of $\mathbb{F} Q$.

Theorem 3.5 [18, Theorem 3.8]. Let $A$ be a finite dimensional algebra and $M$ a basic $\tau$ rigid A-module. Let $T_{M}$ be the Bongartz completion of $M$ in $\bmod A$. Let $E_{M}=\operatorname{End}_{A}\left(T_{M}\right)$ and $D_{M}=E_{M} /\left\langle e_{M}\right\rangle$, where $e_{M}$ is the idempotent corresponding to the projective $E_{M}$ module $\operatorname{Hom}_{A}\left(T_{M}, M\right)$. Then there is an additive exact equivalence of categories between the category $J(M)$, (the $\tau$-perpendicular category of $M$ in $\bmod A)$ and the category $\bmod D_{M}$. Moreover, if $M$ is indecomposable we have that $\delta\left(D_{M}\right)=\delta(A)-1$.

We now prove some results which will be crucial in our strategy for calculating the number of $\tau$-exceptional sequences in $\bmod A$.

Definition 3.6 Interleaving. Let $X=\left(X_{1}, X_{2}, \ldots, X_{s}\right)$ and $Y=\left(Y_{1}, Y_{2}, \ldots, Y_{t}\right)$ be sequences. An interleaved sequence of $X$ and $Y$ is a sequence $Z=\left(Z_{1}, Z_{2}, \ldots, Z_{s+t}\right)$ with 
$Z_{i} \in\left\{X_{j}: 1 \leq i \leq s\right\} \cup\left\{Y_{j}: 1 \leq j \leq t\right\}$ such that the subsequence of $Z$ containing only elements $X$ or $Y$ is precisely $X$ or $Y$ respectively.

Example 3.7 Let $X=\left(5, \frac{4}{5}, 6\right)$ and $Y=\left(2, \frac{1}{2}\right)$ be sequences in $\bmod \Gamma_{6}^{2}$. The sequence $Z=\left(2,5, \frac{4}{5}, \frac{1}{2}, 6\right)$ is an interleaved sequence of $X$ and $Y$. However $W=\left(\begin{array}{l}4 \\ 5\end{array}, 5,2,6, \frac{1}{2}\right)$ is not an interleaved sequence of $X$ and $Y$ because the subsequence containing only elements of $X$ is not equal to $X$.

Let $A$ and $B$ be finite-dimensional $\mathbb{F}$-algebras and let $\bmod A$ and $\bmod B$ be the categories of finitely generated left $A$-modules and left $B$-modules respectively. We may consider the category $\bmod A \oplus \bmod B$, the direct product category of $\bmod A$ and $\bmod B$. The objects of $\bmod A \oplus \bmod B$ are pairs $(M, N)$ with $M \in \bmod A$ and $N \in \bmod B$. A morphism between a pair of objects, $\left(M_{1}, N_{1}\right)$ and $\left(M_{2}, N_{2}\right)$ in $\bmod A \oplus \bmod B$ is a pair of morphisms $(f$ : $M_{1} \rightarrow M_{2}, g: N_{1} \rightarrow N_{2}$ ) where $f \in \bmod A$ and $g \in \bmod B$. The indecomposable objects of $\bmod A \oplus \bmod B$ are pairs $(M, 0)$ and $(0, N)$ where $M$ and $N$ are indecomposable in their respective categories. The category $\bmod A \oplus \bmod B$ is an abelian category, in fact, there is an exact, additive equivalence to $\bmod (A \times B)$. The category $\bmod A \oplus \bmod B$ also has an Auslander-Reiten translate $\tau_{A, B}$ which acts in the obvious way i.e. $\tau_{A, B}(M, 0)=\left(\tau_{A} M, 0\right)$ and $\tau_{A, B}(0, N)=\left(0, \tau_{B} N\right)$. It is easy to see that the above exact equivalence preserves the Auslander-Reiten translations, since irreducible morphisms, left minimal almost split and right minimal almost split morphisms are preserved under equivalence of categories. Let $M$ be an $A$-module, we identify $M$ with the object $(M, 0)$ in $\bmod A \oplus \bmod B$. We like wise identify the $B$-module $N$ with the object $(0, N)$ in $\bmod A \oplus \bmod B$. It is easy to observe that $(M, 0)$ is $\tau$-rigid in $\bmod A \oplus \bmod B$ if and only if $M$ is $\tau$-rigid in $\bmod A$. The similar statement for $(0, N)$ and $N$ is also true.

Theorem 3.8 Let $A$ and $B$ be finite dimensional $\mathbb{F}$-algebras. Suppose $X=$ $\left(X_{1}, X_{2}, \ldots, X_{s}\right)$ is a $\tau$-exceptional sequence in $\bmod A$ and $Y=\left(Y_{1}, Y_{2}, \ldots, Y_{t}\right)$ is a $\tau$-exceptional sequence in mod $B$. Suppose $Z=\left(Z_{1}, Z_{2}, \ldots, Z_{s+t}\right)$ is an interleaved sequence of $X$ and $Y$. Then $Z$ is a $\tau$-exceptional sequence in $\bmod A \oplus \bmod B$.

Proof We prove this by induction on $s+t$. For the base case, suppose $s+t=1$. Without loss of generality suppose $t=0$, so $Z=\left(X_{1}\right)$. By assumption, $X_{1}$ is $\tau$-rigid in $\bmod A$, so it is $\tau$-rigid in $\bmod A \oplus \bmod B$. This completes the base case.

Suppose the statement is true for $s+t=m$. We consider the $s+t=m+1$ case. Suppose the sequence $Z=\left(Z_{1}, Z_{2}, \ldots, Z_{m+1}\right)$ is an interleaved sequence of $X=\left(X_{1}, X_{2}, \ldots, X_{s}\right)$ and $Y=\left(Y_{1}, Y_{2}, \ldots, Y_{t}\right)$, where $X$ is a $\tau$-exceptional sequence in $\bmod A$ and $Y$ is a $\tau$ exceptional sequence in $\bmod B$. Suppose without loss of generality that $Z_{m+1}$ is in $X$ i.e. $Z_{m+1}=X_{s}$. To show that $Z$ is a $\tau$-exceptional sequence in $\bmod A \oplus \bmod B$, we need to show that $Z_{m+1}$ is $\tau$-rigid in $\bmod A \oplus \bmod B$ and that $\left(Z_{1}, Z_{2}, \ldots, Z_{m}\right)$ is a $\tau$-exceptional sequence in $J_{(A, B)}\left(Z_{m+1}\right)$, the $\tau$-perpendicular category of $Z_{m+1}$ in $\bmod A \oplus \bmod B$. By assumption, $Z_{m+1}$ is $\tau$-rigid in $\bmod A$, so it is $\tau$-rigid in $\bmod A \oplus \bmod B$. Observe that $\operatorname{Hom}_{\bmod A \oplus \bmod B}\left(X_{s}, N\right)=\operatorname{Hom}_{\bmod A \oplus \bmod B}\left(N, \tau X_{s}\right)=0$ for all $N \in \bmod B$, so it follows that

$$
\begin{gathered}
J_{(A, B)}\left(Z_{m+1}\right)=\{U \in \bmod A \oplus \bmod B \\
\left.: \operatorname{Hom}_{\bmod A \oplus \bmod B}\left(X_{s}, U\right)=\operatorname{Hom}_{\bmod A \oplus \bmod B}\left(U, \tau_{A} X_{s}\right)=0\right\} \\
=J_{\bmod A}\left(X_{s}\right) \oplus \bmod B
\end{gathered}
$$


where $J_{\bmod A}\left(X_{S}\right)$ is the $\tau$-perpendicular category of $X_{S}$ in $\bmod A$. By Theorem 3.5 , $J_{\bmod A}\left(X_{S}\right)$ is equivalent to a category of modules over some finite dimensional $\mathbb{F}$-algebra. By assumption, $X$ is a $\tau$-exceptional sequence in $\bmod A$, thus $X^{\prime}=\left(X_{1}, X_{2}, \ldots, X_{s-1}\right)$ is a $\tau$-exceptional sequence in $J_{\bmod A}\left(X_{s}\right)$. Moreover, $Z^{\prime}=\left(Z_{1}, Z_{2}, \ldots, Z_{m}\right)$ is an interleaved sequence of $X^{\prime}$ and $Y$, so it follows by the inductive hypothesis that $Z^{\prime}$ is a $\tau$-exceptional sequence in $J_{\bmod A}\left(X_{s}\right) \oplus \bmod B=J_{(A, B)}\left(Z_{m+1}\right)$, hence $Z$ is a $\tau$-exceptional sequence in $\bmod A \oplus \bmod B$. This completes the proof.

We now prove the converse statement.

Theorem 3.9 Let $A$ and $B$ be finite dimensional $\mathbb{F}$-algebras. Suppose $Z=$ $\left(Z_{1}, Z_{2}, \ldots, Z_{m}\right)$ is a $\tau$-exceptional sequence in $\bmod A \oplus \bmod B$. Then $Z$ is an interleaved sequence of some $X=\left(X_{1}, X_{2}, \ldots, X_{s}\right)$ and $Y=\left(Y_{1}, Y_{2}, \ldots, Y_{m-s}\right)$, where $X$ is a $\tau$-exceptional sequence in mod $A$ and $Y$ is a $\tau$-exceptional sequence in mod $B$.

Proof We prove this by induction on $m$.

For the base case, suppose $m=1$, so $Z=\left(Z_{1}\right)$ is a $\tau$-exceptional sequence in $\bmod A \oplus$ $\bmod B$. The module $Z_{1}$ either lies in $\bmod A$ or $\bmod B$. Suppose without loss of generality that $Z_{1} \in \bmod A$. So we define the sequence $X:=\left(Z_{1}\right)$ and the sequence $Y$ to be the empty sequence. The sequence $Z$ is trivially an interleaved sequence of $X$ and $Y$. As $Z$ is a $\tau$-exceptional sequence in $\bmod A \oplus \bmod B$, by definition $Z_{1}$ is $\tau$-rigid in $\bmod A \oplus \bmod B$, so $Z_{1}$ is $\tau$-rigid in mod $A$. This completes the base case.

Now suppose the statement is true for $m=k$. We consider the $m=k+1$ case. The sequence $Z=\left(Z_{1}, Z_{2}, \ldots, Z_{k+1}\right)$ is a $\tau$-exceptional sequence in $\bmod A \oplus \bmod B$, so by definition, $Z_{k+1}$ is $\tau$-rigid in $\bmod A \oplus \bmod B$ and the sequence $Z^{\prime}=\left(Z_{1}, Z_{2}, \ldots, Z_{k}\right)$ is a $\tau$-exceptional sequence in $J_{(A, B)}\left(Z_{k+1}\right)$, the $\tau$-perpendicular category of $Z_{k+1}$ in $\bmod A \oplus \bmod B$. Suppose without loss of generality that $Z_{k+1} \in \bmod A$. We then observe that $\operatorname{Hom}_{\bmod } A \oplus \bmod B\left(Z_{k+1}, N\right)=\operatorname{Hom}_{\bmod A \oplus \bmod B}\left(N, \tau Z_{k+1}\right)=0$ for all $N \in \bmod B$, so it follows that

$$
\begin{gathered}
J_{(A, B)}\left(Z_{m+1}\right)=\left\{U \in \bmod A \oplus \bmod B: \operatorname{Hom}_{\bmod A \oplus \bmod B}\left(X_{s}, U\right)=\operatorname{Hom}_{\bmod A \oplus \bmod B}\left(U, \tau_{A} X_{s}\right)=0\right\} \\
=J_{\bmod A}\left(Z_{k+1}\right) \oplus \bmod B,
\end{gathered}
$$

where $J_{\bmod A}\left(Z_{k+1}\right)$ is the $\tau$-perpendicular category of $Z_{k+1}$ in $\bmod A$. By theorem 3.5 we have that $J_{\bmod A}\left(Z_{k+1}\right)$ is equivalent to a category of modules over some finite dimensional $\mathbb{F}$-algebra. So we may apply the inductive hypothesis to $Z^{\prime}$, hence $Z^{\prime}$ is an interleaved sequence of some $X^{\prime}=\left(X_{1}, X_{2}, \ldots, X_{s}\right)$ and $Y=\left(Y_{1}, Y_{2}, \ldots, Y_{k-s}\right)$, where $X^{\prime}$ is a $\tau$-exceptional sequence in $J_{\bmod A}\left(Z_{k+1}\right)$ and $Y$ is a $\tau$-exceptional sequence in $\bmod B$. Since $Z_{k+1}$ is $\tau$-rigid in $\bmod A \oplus \bmod B$, it is also $\tau$-rigid $\bmod A$, hence $X=$ $\left(X_{1}, X_{2}, \ldots, X_{s}, Z_{k+1}\right)$ is a $\tau$-exceptional sequence in mod $A$. Clearly $Z$ is an interleaved sequence $X$ and $Y$, so this completes the proof by induction.

We will now recall some standard definitions from [5] which we require for the rest of this paper. Recall that the radical of an $A$-module $M$, denoted by $\operatorname{rad}(M)$, is defined to be the intersection of all maximal submodules of $M$. The quotient $M / \operatorname{rad}(M)$ is known as the top of $M$ and is denoted top $(M)$. The socle of an $A$-module $M$ denoted $\operatorname{soc}(M)$ is the sum of the simple submodules of $M$. 
Definition 3.10 Radical Series [5, V.1]. Let $M$ be an $A$-module. The radical series of $M$ is defined to be the following sequence of submodules,

$$
0 \subset \cdots \subset \operatorname{rad}^{2}(M) \subset \operatorname{rad}(M) \subset M .
$$

Since the left $A$-modules $M$ are finite dimensional as $\mathbb{F}$-vector spaces, there exists a least positive integer $m$ such that $\operatorname{rad}^{m}(M)=0$. The integer $m$ is called the length of the radical series and we denote it by $l(M)=m$. We will also refer to $l(M)$ as the length of the module $M$.

Proposition 3.11 [5, V.3.5, V.4.1, V.4.2]. Let A be a basic connected Nakayama algebra and let $M$ be an indecomposable A-module. Then there exists some $1 \leq i \leq n$ and $1 \leq j \leq$ $l\left(P_{i}\right)$, such that $M \cong P_{i} / \mathrm{rad}^{j}\left(P_{i}\right)$ and $j=l(M)$. Moreover, if $M$ is not projective, we have that $\tau M \cong \operatorname{rad}\left(P_{i}\right) / \mathrm{rad}^{j+1}\left(P_{i}\right)$ and $l(\tau M)=l(M)$.

So we see that modules $M$ of Nakayama algebras are uniquely determined by their top, top $(M)$ and their length $l(M)$.

Proposition 3.12 [1, Lemma 2.4]. Let $M=P_{j} / \operatorname{rad}^{l}\left(P_{j}\right)$ and $N=P_{i} / \operatorname{rad}^{k}\left(P_{i}\right)$ for $1 \leq$ $i, j, k, l \leq n$. Then the following conditions are equivalent,

1. $\operatorname{Hom}(M, N) \neq 0$

2. $j \in[i,(i+k-1)]_{n}$ and $(i+k-1)_{n} \in[j,(j+l-1)]_{n}$

\section{The $\Gamma_{n}^{2}$ Case}

Let $n \geq 1$ be a positive integer. In this section we will derive a closed formula for the number of complete $\tau$-exceptional sequences in $\bmod \Gamma_{n}^{2}$. Recall that we denote by $A_{n}$ the linearly oriented quiver with $n$ vertices,

$$
1 \stackrel{\alpha_{1}}{\longrightarrow} 2 \stackrel{\alpha_{2}}{\longrightarrow} 3 \stackrel{\alpha_{3}}{\longrightarrow} \ldots \stackrel{\alpha_{n-2}}{\longrightarrow} n-1 \stackrel{\alpha_{n-1}}{\longrightarrow} n .
$$

The algebra $\Gamma_{n}^{2}$ is defined to be the $\mathbb{F}$-algebra, $\mathbb{F} A_{n} / R_{Q}^{2}$. This is the path algebra of the quiver $A_{n}$ modulo the relations $\alpha_{i} \alpha_{i+1}=0$ for $1 \leq i \leq n-2$.

The category $\bmod \Gamma_{n}^{2}$ has the following Auslander-Reiten quiver.
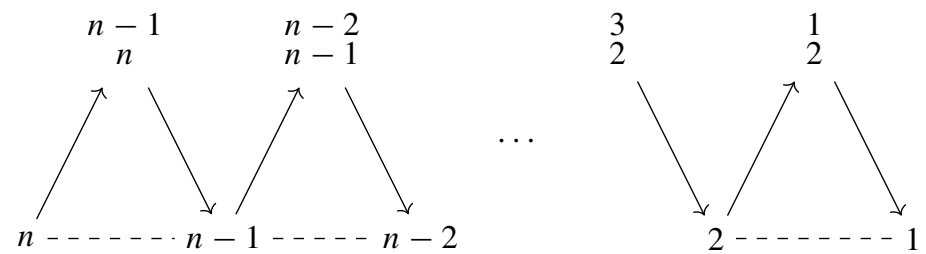

Our strategy for calculating the number of $\tau$-exceptional sequences is straightforward. For each $M$ in $\operatorname{ind}\left(\bmod \Gamma_{n}^{2}\right)$, we will calculate the number of complete $\tau$-exceptional sequences ending in $M$. If $M$ is indecomposable, then either $M=P_{i}$, the projective at vertex $i$ of $A_{n}$, or $M=S_{i}$, the simple at vertex $i$ of $A_{n}$ (notice that $S_{n}=P_{n}$ ). In the former case $\tau P_{i}=0$ for $1 \leq i \leq n$ and in the latter case $\tau S_{j}=S_{j+1}$ for $1 \leq j \leq n-1$. In both cases we see that $M$ is $\tau$-rigid i.e. every indecomposable $M$ in $\bmod \Gamma_{n}^{2}$ is $\tau$-rigid. We recall that a sequence of indecomposable modules $\left(M_{1}, M_{2}, \ldots, M_{n-1}, M\right)$ is a $\tau$-exceptional sequence in $\bmod \Gamma_{n}^{2}$ if $M$ is $\tau$-rigid, and $\left(M_{1}, M_{2}, \ldots, M_{n-1}\right)$ is a $\tau$-exceptional sequence in $J(M)$. 
Having seen that every indecomposable module $M$ is $\tau$-rigid, what is left to do is to calculate $J(M)$ for each indecomposable module. Theorem 3.5 and Lemma 3.4 are the main tools for these calculations.

Proposition 4.1 Let $P_{i}$ be an indecomposable projective module in $\bmod \Gamma_{n}^{2}$ for some $1 \leq$ $i \leq n$. Then the $\tau$-perpendicular category of $P_{i}$ in $\bmod \Gamma_{n}^{2}$ is $J\left(P_{i}\right) \cong \bmod \Gamma_{i-1}^{2} \oplus \bmod \Gamma_{n-i}^{2}$.

Proof By definition $T_{P_{i}}=\mathcal{P}\left({ }^{\perp}\left(\tau P_{i}\right)\right)$. Since $\tau P_{i}=0$, we have that ${ }^{\perp}\left(\tau P_{i}\right)=\bmod \Gamma_{n}^{2}$. As a result the Ext-projectives of ${ }^{\perp}\left(\tau P_{i}\right)$ are just the projectives of $\bmod \Gamma_{n}^{2}$, hence

$$
T_{P_{i}}=\mathcal{P}\left({ }^{\perp}\left(\tau P_{i}\right)\right)=\bigoplus_{j=1}^{n} P_{j} .
$$

Thus the $\mathbb{F}$-algebra $E_{P_{i}}=\operatorname{End}_{\Gamma_{n}^{2}}\left(T_{P_{i}}\right)$ is precisely given by the path algebra of $A_{n}^{\text {op }}$,

$$
1 \stackrel{\alpha_{2}}{\longleftarrow} 2 \stackrel{\alpha_{3}}{\longleftarrow} 3 \stackrel{\alpha_{4}}{\longleftarrow} \ldots \stackrel{\alpha_{i-1}}{\longleftarrow} i-1 \stackrel{\alpha_{i}}{\longleftarrow} i \stackrel{\alpha_{i+1}}{\longleftarrow} i+1 \stackrel{\alpha_{i+2}}{\longleftarrow} \ldots \stackrel{\alpha_{n-1}}{\longleftarrow} n-1 \stackrel{\alpha_{n}}{\longleftarrow} n
$$

modulo the relations $\alpha_{j} \alpha_{j-1}=0$ for $3 \leq j \leq n$. Let $A_{n}^{\text {op }(i)}$ be the quiver obtained from $A_{n}^{\mathrm{op}}$ by removing the vertex $i$ and any arrows incident to $i$,

$$
1 \stackrel{\alpha_{2}}{\longleftarrow} 2 \stackrel{\alpha_{3}}{\longleftarrow} 3 \stackrel{\alpha_{4}}{\longleftarrow} \ldots \stackrel{\alpha_{i-1}}{\longleftarrow} i-1 \quad i+1 \stackrel{\alpha_{i+2}}{\longleftarrow} \ldots \stackrel{\alpha_{n-1}}{\longleftarrow} n-1 \stackrel{\alpha_{n}}{\longleftarrow} n .
$$

The quiver $A_{n}^{\mathrm{op}(i)}$ has relations $\alpha_{j} \alpha_{j-1}=0$ for $3 \leq j \leq i-1$ and $i+3 \leq j \leq n$. By Lemma 3.4, $D_{P_{i}}=E_{P_{i}} /\left\langle e_{P_{i}}\right\rangle$ is the path algebra of $A_{n}^{\mathrm{op}(i)}$ modulo its relations. So it follows that $J\left(P_{i}\right) \cong \bmod \Gamma_{i-1}^{2} \oplus \bmod \Gamma_{n-i}^{2}$ by Theorem 3.5 .

Proposition 4.2 Let $S_{i}$ be a simple non-projective module in $\bmod \Gamma_{n}^{2}$ for some $1 \leq i \leq n-$ 1. Then the $\tau$-perpendicular category of $S_{i}$ in $\bmod \Gamma_{n}^{2}$ is $J\left(S_{i}\right) \cong \bmod \Gamma_{i-1}^{2} \oplus \bmod \Gamma_{n-i-1}^{2} \oplus$ $\bmod \Gamma_{1}^{2}$.

Proof By definition $T_{S_{i}}=\mathcal{P}\left({ }^{\perp}\left(\tau S_{i}\right)\right)$. Since $S_{i}$ is a simple non-projective indecomposable module $S_{i}$, we have that $\tau S_{i}=S_{i+1}$. Note that the only indecomposable $\Gamma_{n}^{2}$-modules not in ${ }^{\perp}\left(\tau S_{i}\right)$ are $S_{i+1}$ and $P_{i+1}$. Observe also that $\operatorname{Ext}_{\Gamma_{n}^{2}}\left(P_{j},{ }^{\perp}\left(\tau S_{i}\right)\right)=0$ if $j \neq i+1,1 \leq j \leq n$. We also have that $\operatorname{Ext}_{\Gamma_{n}^{2}}\left(S_{j},{ }^{\perp}\left(\tau S_{i}\right)\right) \neq 0$ for $j \neq i$, and $1 \leq j \leq n$ because $S_{j+1}$ is in ${ }^{\perp}\left(\tau S_{i}\right)$ in these cases. By Proposition 3.1, $S_{i}$ is Ext-projective in ${ }^{\perp}\left(\tau S_{i}\right)$. Therefore

$$
T_{S_{i}}=\mathcal{P}\left({ }^{\perp}\left(\tau S_{i}\right)\right)=S_{i} \oplus \bigoplus_{j \neq i+1} P_{j} .
$$

The $\mathbb{F}$-algebra $E_{S_{i}}=\operatorname{End}_{\Gamma_{n}^{2}}\left(T_{S_{i}}\right)$ is the path algebra of the following quiver,

$$
1 \stackrel{\alpha_{2}}{\longleftarrow} 2 \stackrel{\alpha_{3}}{\longleftarrow} \ldots \stackrel{\alpha_{i-1}}{\longleftarrow} i-1 \stackrel{\alpha_{v S_{i}}}{\longleftarrow} v_{S_{i}} \stackrel{\alpha_{i}}{\longleftarrow} i \quad i+2 \stackrel{\alpha_{i+3}}{\longleftarrow} \ldots \stackrel{\alpha_{n-1}}{\longleftarrow} n-1 \stackrel{\alpha_{n}}{\longleftarrow} n
$$

modulo the relations $\alpha_{j} \alpha_{j-1}=0$ for $3 \leq j \leq i-1$ and $i+4 \leq j \leq n$. Here the vertex $v_{S_{i}}$ is the one corresponding to the simple non-projective module $S_{i}$ and the rest correspond to the projective modules $P_{j}$. Consider the following quiver obtained from the one above by removing the vertex $v_{S_{i}}$ and any arrows incident to $v_{S_{i}}$,

$$
1 \stackrel{\alpha_{2}}{\longleftarrow} 2 \stackrel{\alpha_{3}}{\longleftarrow} \ldots \stackrel{\alpha_{i-1}}{\longleftarrow} i-1 \quad i \quad i+2 \stackrel{\alpha_{i+3}}{\longleftarrow} \ldots \stackrel{\alpha_{n-1}}{\longleftarrow} n-1 \stackrel{\alpha_{n}}{\longleftarrow} n,
$$

it has the relations $\alpha_{j} \alpha_{j-1}=0$ for $3 \leq j \leq i-1$ and $i+4 \leq j \leq n$. By Lemma 3.4, $D_{S_{i}}=E_{S_{i}} /\left\langle e_{S_{i}}\right\rangle$ is the path algebra of this quiver modulo its relations. So it follows that $\bmod D_{S_{i}} \cong \bmod \Gamma_{i-1}^{2} \oplus \bmod \Gamma_{n-i-1}^{2} \oplus \bmod \Gamma_{1}^{2}$. By Theorem 3.5, the statement of this Proposition follows.

Let us denote by $G_{n}$ the number of complete $\tau$-exceptional sequences of $\bmod \Gamma_{n}^{2}$. When $n=0,1,2$ the $\tau$-exceptional sequences coincide with the "classical" exceptional sequences 
since the algebra $\Gamma_{n}^{2}$ is the hereditary Dynkin type A algebra $\mathbb{A}_{n}$ in this case. Hence, $G_{0}=$ $G_{1}=1$ and $G_{2}=3$.

Lemma 4.3 Let $P_{i}$ be the indecomposable projective module in $\bmod \Gamma_{n}^{2}$ at the vertex $i$ of $A_{n}$ for some $1 \leq i \leq n$. The number of complete $\tau$-exceptional sequences in $\bmod \Gamma_{n}^{2}$ ending in $P_{i}$ is,

$$
\left(\begin{array}{c}
n-1 \\
n-i, i-1
\end{array}\right) G_{n-i} G_{i-1}
$$

Proof Let $\left(X_{1}, X_{2}, \ldots, X_{n-1}, P_{i}\right)$ be a complete $\tau$-exceptional sequence in $\bmod \Gamma_{n}^{2}$ ending in $P_{i}$. Then by definition and the fact that $\delta\left(J\left(P_{i}\right)\right)=n-1$, the sequence $\left(X_{1}, X_{2}, \ldots, X_{n-1}\right)$ is a $\tau$-exceptional sequence in $J\left(P_{i}\right)$. So to count the number of complete $\tau$-exceptional sequences in $\bmod \Gamma_{n}^{2}$ ending in $P_{i}$, we just need to count the number of complete $\tau$-exceptional sequences in $J\left(P_{i}\right)$. By Lemma $4.1, J\left(P_{i}\right) \cong \bmod \Gamma_{i-1}^{2} \oplus$ $\bmod \Gamma_{n-i}^{2}$. By Theorem 3.8 and 3.9, the $\tau$-exceptional sequences of $J\left(P_{i}\right)$ are interleavings of $\tau$-exceptional sequences of $\bmod \Gamma_{i-1}^{2}$ and $\bmod \Gamma_{n-i}^{2}$. The number of interleaved sequences coming from a sequence of length $i-1$ and a sequence of length $n-i$ is precisely $\left(\begin{array}{c}n-1 \\ n-i, i-1\end{array}\right)$. Thus the number of complete $\tau$-exceptional sequences ending in $P_{i}$ is $\left(\begin{array}{c}n-1 \\ n-i, i-1\end{array}\right) G_{n-i} G_{i-1}$.

Lemma 4.4 Let $S_{i}$ be the indecomposable simple non-projective module in $\bmod \Gamma_{n}^{2}$ at the vertex $i$ of $A_{n}$ for some $1 \leq i \leq n-1$. The number of $\tau$-exceptional sequences in $\bmod \Gamma_{n}^{2}$ ending in $S_{i}$ is,

$$
\left(\begin{array}{c}
n-1 \\
n-i-1, i-1
\end{array}\right) G_{n-i-1} G_{i-1}
$$

Proof Let $\left(X_{1}, X_{2}, \ldots, X_{n-1}, S_{i}\right)$ be a complete $\tau$-exceptional sequence in $\bmod \Gamma_{n}^{2}$ ending in $S_{i}$. Then by definition and the fact that $\delta\left(J\left(S_{i}\right)\right)=n-1$, the sequence $\left(X_{1}, X_{2}, \ldots, X_{n-1}\right)$ is a complete $\tau$-exceptional sequence in $J\left(S_{i}\right)$. Hence to count the number of complete $\tau$-exceptional sequences in $\bmod \Gamma_{n}^{2}$ ending in $S_{i}$, we just need to count the number of complete $\tau$-exceptional sequences in $J\left(S_{i}\right)$. By Lemma 4.2, $J\left(S_{i}\right) \cong$ $\bmod \Gamma_{i-1}^{2} \oplus \bmod \Gamma_{n-i-1}^{2} \oplus \bmod \Gamma_{1}^{2}$. The number of interleaved sequences coming from a sequence of length $i-1$, a sequence of length $n-i-1$ and a sequence of length 1 is precisely $\left(\begin{array}{c}n-1 \\ n-i-1, i-1,1\end{array}\right)=\left(\begin{array}{c}n-1 \\ n-i-1, i-1\end{array}\right)$. Thus the number of complete $\tau$-exceptional sequences ending in $S_{i}$ is $\left(\begin{array}{c}n-1 \\ n-i-1, i-1\end{array}\right) G_{n-i-1} G_{i-1}$.

Theorem 4.5 Let $G_{n}$ denote the number of complete $\tau$-exceptional sequences in $\bmod \Gamma_{n}^{2}$. Then $G_{n}$ satisfies the recurrence relation,

$$
G_{n}=\sum_{i=1}^{n}\left(\begin{array}{c}
n-1 \\
n-i, i-1
\end{array}\right) G_{n-i} G_{i-1}+\sum_{i=1}^{n-1}\left(\begin{array}{c}
n-1 \\
n-i-1, i-1
\end{array}\right) G_{n-i-1} G_{i-1},
$$

with initial conditions $G_{0}=G_{1}=1$.

Proof Let $M$ be an indecomposable in $\bmod \Gamma_{n}^{2}$, then either $M$ is projective or $M$ simple nonprojective. There are $n$ projective indecomposable modules in mod $\Gamma_{n}^{2}$ denoted by $P_{i}$ for $1 \leq$ $i \leq n$. There are $n-1$ simple non-projective indecomposable modules in $\bmod \Gamma_{n}^{2}$ denoted by 
$S_{i}$ for $1 \leq i \leq n-1$. Therefore by Lemma 4.3 and 4.4, $G_{n}=\sum_{i=1}^{n}\left(\begin{array}{c}n-1 \\ n-i, i-1\end{array}\right) G_{n-i} G_{i-1}+$ $\sum_{i=1}^{n-1}\left(\begin{array}{c}n-1 \\ n-i-1, i-1\end{array}\right) G_{n-i-1} G_{i-1}$.

Theorem 4.5 allows us to calculate the first ten terms of the sequence $\left(G_{n}\right)_{n=0}^{\infty}$ as:

$$
1,1,3,12,66,450,3690,35280,385560,4740120,6475140 .
$$

An ordered set partition of $\{1,2, \ldots, n\}$ is a partition of the set $\{1,2, \ldots, n\}$ together with a total order on the sets in the partition. We refer to the sets in an ordered partition as blocks. The restricted Fubini number $F_{n, \leqslant m}$ counts the number of ordered set partitions of $\{1,2, \ldots, n\}$ with blocks of size at most $m$. The restricted Stirling number of the second kind, denoted by $\left\{\begin{array}{l}n \\ k\end{array}\right\}_{\leqslant m}$, is the number of (unordered) partitions of $\{1,2, \ldots, n\}$ into $k$ subsets with the restriction that each block contains at most $m$ elements. Therefore

$$
F_{n, \leqslant m}=\sum_{k=0}^{n} k !\left\{\begin{array}{l}
n \\
k
\end{array}\right\}_{\leqslant m} .
$$

It is shown in [21, Section 5.4] that the restricted Fubini numbers satisfy the recurrence:

$$
F_{n, \leqslant m}=\sum_{l=1}^{m}\left(\begin{array}{l}
n \\
l
\end{array}\right) F_{n-l, \leqslant m} .
$$

The sequence $\left(F_{n, \leqslant 2}\right)$ is listed on the On-line Encyclopedia of Integer Sequences (OEIS) as the sequence A080599. The first terms of this sequence coincide with the first terms we calculated for $\left(G_{n}\right)$ so we would like to prove that it is the case that $F_{n, \leqslant 2}=G_{n}$.

When $m=2$ the recurrence for $F_{n, \leqslant m}$ is given as $F_{n, \leqslant 2}=n F_{n-1, \leqslant 2}+\left(\begin{array}{c}n \\ 2\end{array}\right) F_{n-2, \leqslant 2}$. In the paper [13, Theorem 3.7], the authors derive the closed formula

$$
F_{n, \leqslant 2}=\frac{n !}{\sqrt{3}}\left((\sqrt{3}-1)^{-n-1}-(-\sqrt{3}-1)^{-n-1}\right) .
$$

An exponential generating function for $F_{n, \leqslant m}$ is given in [20, Theorem 4]:

$$
\sum_{n=0}^{\infty} F_{n, \leqslant m} \frac{x^{n}}{n !}=\frac{1}{1-x-\frac{x^{2}}{2 !}-\ldots \frac{x^{m}}{m !}} .
$$

We will show that $G_{n}=F_{n, \leqslant 2}$ by showing that the exponential generating functions for $G_{n}$ and $F_{n, \leqslant 2}$ coincide.

Theorem 4.6 Let $G_{n}$ denote the number of complete $\tau$-exceptional sequences in mod $\Gamma_{n}^{2}$. The exponential generating function of $G_{n}$ is as follows,

$$
\sum_{n=0}^{\infty} G_{n} \frac{x^{n}}{n !}=\frac{1}{1-x-\frac{x^{2}}{2 !}}
$$

Therefore $G_{n}=F_{n, \leqslant 2}$ and

$$
G_{n}=\frac{n !}{\sqrt{3}}\left((\sqrt{3}-1)^{-n-1}-(-\sqrt{3}-1)^{-n-1}\right) .
$$

Proof First let us recall the recurrence relation for $G_{n}$.

$$
G_{n}=\sum_{i=1}^{n}\left(\begin{array}{c}
n-1 \\
n-i, i-1
\end{array}\right) G_{n-i} G_{i-1}+\sum_{i=1}^{n-1}\left(\begin{array}{c}
n-1 \\
n-i-1, i-1
\end{array}\right) G_{n-i-1} G_{i-1} .
$$




$$
=\sum_{i=1}^{n} \frac{(n-1) !}{(n-i) !(i-1) !} G_{n-i} G_{i-1}+\sum_{i=1}^{n-1} \frac{(n-1) !}{(n-i-1) !(i-1) !} G_{n-i-1} G_{i-1} .
$$

Therefore

$$
G_{n+1}=\sum_{i=1}^{n+1} \frac{n !}{(n+1-i) !(i-1) !} G_{n+1-i} G_{i-1}+\sum_{i=1}^{n} \frac{n !}{(n-i) !(i-1) !} G_{n-i} G_{i-1} .
$$

Let

$$
g(x)=\sum_{n=0}^{\infty} G_{n} \frac{x^{n}}{n !} \text { with } g(0)=1,
$$

be the exponential generating function of $G_{n}$. We then have that the first derivative of $g(x)$ is $g^{\prime}(x)=\sum_{n=0}^{\infty} G_{n+1} \frac{x^{n}}{n !}$. Expanding $G_{n+1}$ in $g^{\prime}(x)$ by the recurrence relation above we obtain the following.

$$
\begin{gathered}
g^{\prime}(x)=\sum_{n=0}^{\infty}\left(\sum_{i=1}^{n+1} \frac{n !}{(n+1-i) !(i-1) !} G_{n+1-i} G_{i-1}\right) \frac{x^{n}}{n !}+\sum_{n=0}^{\infty}\left(\sum_{i=1}^{n} \frac{n !}{(n-i) !(i-1) !} G_{n-i} G_{i-1}\right) \frac{x^{n}}{n !} \\
=\sum_{n=0}^{\infty}\left(\sum_{i=1}^{n+1} \frac{G_{n+1-i} G_{i-1}}{(n+1-i) !(i-1) !}\right) x^{n}+\sum_{n=0}^{\infty}\left(\sum_{i=1}^{n} \frac{G_{n-i} G_{i-1}}{(n-i) !(i-1) !}\right) x^{n} .
\end{gathered}
$$

Recall the Cauchy product of formal power series is as follows,

$$
\left(\sum_{s=0}^{\infty} a_{s} x^{s}\right)\left(\sum_{t=0}^{\infty} b_{t} x^{t}\right)=\sum_{k=0}^{\infty} c_{k} x^{k} \text { where } c_{k}=\sum_{l=0}^{k} a_{l} b_{k-l} .
$$

By performing a change of variable in $g^{\prime}(x)$ by setting $j=i-1$ and factorising $x$ from the right summand we write,

$$
g^{\prime}(x)=\sum_{n=0}^{\infty}\left(\sum_{j=0}^{n} \frac{G_{n-j} G_{j}}{(n-j) ! j !}\right) x^{n}+x \sum_{n=0}^{\infty}\left(\sum_{j=0}^{n-1} \frac{G_{n-j-1} G j}{(n-j-1) ! j !}\right) x^{n-1} .
$$

Using the Cauchy product of formal power series, we obtain the following first order nonlinear ordinary differential equation.

$$
g^{\prime}(x)=(g(x))^{2}+x(g(x))^{2}=(1+x)(g(x))^{2} \text { with initial conditions } g(0)=1 .
$$

It is easy to check that the unique solution to this ODE is given by,

$$
g(x)=\frac{-2}{-2+x(x+2)}=\frac{1}{1-x-\frac{x^{2}}{2}} .
$$

This completes the proof.

Remark 4.7 Here we focused on $\tau$-exceptional sequences, but it's natural to ask what is known about the more classical exceptional sequences. It is shown in [29] that the number of complete exceptional sequences of $\bmod \Gamma_{n}^{2}$ are equal to the sum, $\sum_{j=1}^{n}\left(\begin{array}{c}n \\ j\end{array}\right) j^{n-j}$. The first ten terms of the sequence $\left(\sum_{j=1}^{n}\left(\begin{array}{c}n \\ j\end{array}\right) j^{n-j}\right)_{n=1}^{\infty}$ are,

$$
\text { 1, 3, 10, 41, 196, 1057, 6322, 41393, 293608, } 2237921 .
$$

For comparison the number of complete $\tau$-exceptional sequences of $\bmod \Gamma_{n}^{2}$ are given by $G_{n}$, the first ten terms of the sequence $\left(G_{n}\right)_{n=1}^{\infty}$ are,

$$
1,3,12,66,450,3690,35280,385560,4740120,6475140 .
$$




\section{The $\Lambda_{n}^{2}$ Case}

Let $n \geq 1$ be a positive integer. In this section we will derive a closed formula for the number of complete $\tau$-exceptional sequences in $\bmod \Lambda_{n}^{2}$. Recall that we denote by $C_{n}$ the linearly oriented $n$-cycle.

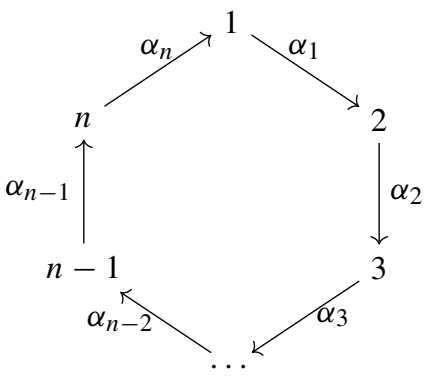

The algebra $\Lambda_{n}^{2}$ is defined to be the $\mathbb{F}$-algebra, $\mathbb{F} C_{n} / R_{Q}^{2}$. This is the path algebra of the quiver $C_{n}$ modulo the relations $\alpha_{j} \alpha_{(j+1)_{n}}=0$ for $1 \leq j \leq n$.

The category $\bmod \Lambda_{n}^{2}$ has the following Auslander-Reiten quiver.
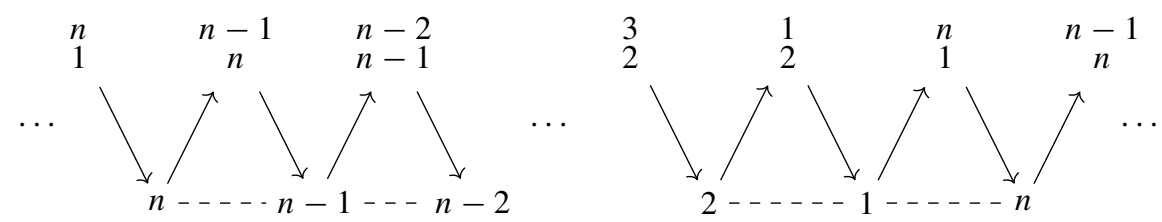

We will use the same approach for calculating the number of complete $\tau$-exceptional sequences for $\bmod \Lambda_{n}^{2}$ as we did for $\bmod \Gamma_{n}^{2}$. If $M$ is indecomposable in $\bmod \Lambda_{n}^{2}$, then $M=P_{i}$, the projective at vertex $i$ of $C_{n}$, or $M=S_{i}$, the simple at vertex $i$ of $C_{n}$. In the former case $\tau P_{i}=0$ and in the latter case $\tau S_{i}=S_{(i+1)_{n}}$. In both cases $M$ is $\tau$-rigid i.e. every indecomposable $M$ in $\bmod \Lambda_{n}^{2}$ is $\tau$-rigid. We recall that a sequence of indecomposable modules $\left(M_{1}, M_{2}, \ldots, M_{n-1}, M\right)$ is a $\tau$-exceptional sequence in $\bmod \Lambda_{n}^{2}$ if $M$ is $\tau$-rigid, and $\left(M_{1}, M_{2}, \ldots, M_{n-1}\right)$ is a $\tau$-exceptional sequence in $J(M)$. Having seen that every indecomposable module is $\tau$-rigid, what is left to do is to calculate $J(M)$ for each indecomposable module. Theorem 3.5 and Lemma 3.4 are the main tools for these calculations.

Proposition 5.1 Let $P_{i}$ be an indecomposable projective module in mod $\Lambda_{n}^{2}$ for some $1 \leq$ $i \leq n$. Then the $\tau$-perpendicular category of $P_{i}$ in $\bmod \Lambda_{n}^{2}$ is $J\left(P_{i}\right) \cong \bmod \Gamma_{n-1}^{2}$.

Proof By definition $T_{P_{i}}=\mathcal{P}\left({ }^{\perp}\left(\tau P_{i}\right)\right)$. Since $P_{i}$ is projective, we have that $\tau P_{i}=0$, therefore ${ }^{\perp}\left(\tau P_{i}\right)=\bmod \Lambda_{n}^{2}$. As a result the Ext-projectives of ${ }^{\perp}\left(\tau P_{i}\right)$ are just the projectives of $\bmod \Lambda_{n}^{2}$, hence

$$
T_{P_{i}}=\mathcal{P}\left({ }^{\perp}\left(\tau P_{i}\right)\right)=\bigoplus_{j=1}^{n} P_{j} .
$$


Thus the $\mathbb{F}$-algebra $E_{P_{i}}=\operatorname{End}_{\Lambda_{n}^{2}}\left(T_{P_{i}}\right)$ is precisely given by the path algebra of the quiver $C_{n}^{\mathrm{op}}$,

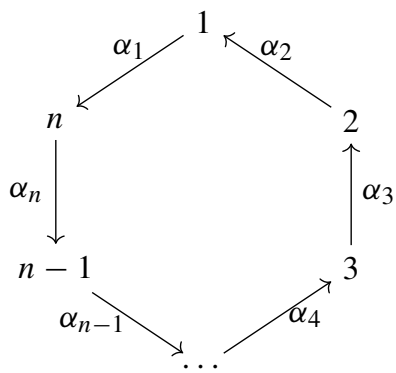

modulo the relations $\alpha_{i} \alpha_{(i-1)_{n}}=0$ for $1 \leq i \leq n$.

Let $C_{n}^{\mathrm{op}(i)}$ be the quiver obtained from $C_{n}^{\mathrm{op}}$ by removing the vertex at $i$ and any arrows incident to $i$.

$i+1 \stackrel{\alpha_{i+2}}{\longleftarrow} i+2 \stackrel{\alpha_{i+3}}{\longleftarrow} \ldots \stackrel{\alpha_{n-1}}{\longleftarrow} n-1 \stackrel{\alpha_{n}}{\longleftarrow} n \stackrel{\alpha_{1}}{\longleftarrow} 1 \stackrel{\alpha_{2}}{\longleftarrow} \ldots \stackrel{\alpha_{i-2}}{\longleftarrow} i-2 \stackrel{\alpha_{i-1}}{\longleftarrow} i-1$

It has the relations $\alpha_{1} \alpha_{n}=0$ and $\alpha_{j} \alpha_{j-1}=0$ for $i+2 \leq j \leq n$ and $2 \leq j \leq$ $i-2$. By Lemma 3.4, $D_{P_{i}}=E_{P_{i}} /\left\langle e_{P_{i}}\right\rangle$ is the path algebra of the quiver $C_{n}^{\text {op }(i)}$ modulo relations. It is easy to see that in fact $D_{P_{i}}$ is isomorphic to $\Gamma_{n-1}^{2}$. Hence by Theorem 3.5, the $\tau$-perpendicular category $J(M) \cong \bmod \Gamma_{n-1}^{2}$.

Proposition 5.2 Let $S_{i}$ be a simple module in $\bmod \Lambda_{n}^{2}$ for some $1 \leq i \leq n$. Then the $\tau$-perpendicular category of $S_{i}$ in $\bmod \Lambda_{n}^{2}$ is $J\left(S_{i}\right) \cong \bmod \Gamma_{n-2}^{2} \oplus \bmod \Gamma_{1}^{2}$.

Proof By definition $T_{S_{i}}=\mathcal{P}\left({ }^{\perp}\left(\tau S_{i}\right)\right)$. Since $S_{i}$ is a simple $\Lambda_{n}^{2}$-module, we have that $\tau S_{i}=$ $S_{(i+1)_{n}}$. Note that the only $\Lambda_{n}^{2}$-modules not in ${ }^{\perp}\left(\tau S_{i}\right)$ are $S_{(i+1)_{n}}$ and $P_{(i+1)_{n}}$. Observe also that $\operatorname{Ext}_{\Lambda_{n}^{2}}\left(P_{j},{ }^{\perp}\left(\tau S_{i}\right)\right)=0$ for $j \neq(i+1)_{n}$ and $1 \leq j \leq n$. However for $j \neq i, i+$ $1, \operatorname{Ext}_{\Lambda_{n}^{2}}\left(S_{j},{ }^{\perp}\left(\tau S_{i}\right)\right) \neq 0$ because $S_{(j+1)_{n}}$ is in ${ }^{\perp}(\tau M)$. By Proposition 3.1, $S_{i}$ is Extprojective in ${ }^{\perp}\left(\tau S_{i}\right)$. Hence

$$
T_{S_{i}}=\mathcal{P}\left({ }^{\perp}\left(\tau S_{i}\right)\right)=S_{i} \oplus \bigoplus_{j \neq(i+1)_{n}} P_{j}
$$

is the Bongartz completion of $S_{i}$.

The $\mathbb{F}$-algebra $E_{S_{i}}=\operatorname{End}_{\Lambda_{n}^{2}}\left(T_{S_{i}}\right)$ is given by the path algebra of the quiver,

$i+2 \stackrel{\alpha_{i+3}}{\longleftarrow} i+3 \stackrel{\alpha_{i+4}}{\longleftarrow} \ldots \stackrel{\alpha_{n-1}}{\longleftarrow} n-1 \stackrel{\alpha_{n}}{\longleftarrow} n \stackrel{\alpha_{1}}{\longleftarrow} 1 \stackrel{\alpha_{2}}{\longleftarrow} \ldots \stackrel{\alpha_{i-1}}{\longleftarrow} i-1 \stackrel{\alpha_{v_{i}}}{\longleftarrow} v_{S_{i}} \stackrel{\alpha i}{\longleftarrow} i$

modulo the relations $\alpha_{v_{S_{i}}} \alpha_{i-1}=0=\alpha_{1} \alpha_{n}$ and $\alpha_{j} \alpha_{j-1}=0$ for $i+4 \leq j \leq n$ and $2 \leq j \leq i-1$. Here the vertex $v_{S_{i}}$ is the one corresponding to the simple module $S_{i}$ and the rest correspond to the projective modules $P_{j}$. By Lemma 3.4, $D_{S_{i}}=E_{S_{i}} /\left\langle e_{S_{i}}\right\rangle$ is the path algebra of the quiver obtained from the one above by removing the vertex $v_{S_{i}}$,

$i+2 \stackrel{\alpha_{i+3}}{\longleftarrow} i+3 \stackrel{\alpha_{i+4}}{\longleftarrow} \ldots \stackrel{\alpha_{n-1}}{\longleftarrow} n-1 \stackrel{\alpha_{n}}{\longleftarrow} \stackrel{\alpha_{1}}{\longleftarrow} 1 \stackrel{\alpha_{2}}{\longleftarrow} \ldots \stackrel{\alpha_{i-1}}{\longleftarrow} i-1$

modulo the relations $\alpha_{1} \alpha_{n}=0$ and $\alpha_{j} \alpha_{j-1}=0$ for $i+4 \leq j \leq n$ and $2 \leq j \leq i-1$. So it follows that $\bmod D_{S_{i}} \cong \bmod \Gamma_{n-2}^{2} \oplus \bmod \Gamma_{1}^{2}$. By Theorem 3.5 the statement of this Proposition follows.

Denote by $L_{n}$ the number of complete $\tau$-exceptional sequences in $\bmod \Lambda_{n}^{2}$. 
Theorem 5.3 Let $L_{n}$ be the number of complete $\tau$-exceptional sequences in $\bmod \Lambda_{n}^{2}$. Then $L_{n}$ satisfies the relation,

$$
L_{n}=n G_{n-1}+n(n-1) G_{n-2},
$$

with initial conditions $L_{1}=1$ and $L_{2}=4$, and where $G_{m}$ denotes the number of complete $\tau$-exceptional sequences in $\bmod \Gamma_{m}^{2}$.

Proof Suppose $M$ is an indecomposable projective $\Lambda_{n}^{2}$-module, then by Lemma 5.1, the $\tau$ perpendicular category $J(M) \cong \bmod \Gamma_{n-1}^{2}$. Suppose $\left(X_{1}, X_{2}, \ldots, X_{n-1}, M\right)$ is a complete $\tau$-exceptional sequence ending in $M$ in $\bmod \Lambda_{n}^{2}$. Then by the fact that $\delta(J(M))=n-1$ and by definition, the sequence $\left(X_{1}, X_{2}, \ldots, X_{n-1}\right)$ is a complete $\tau$-exceptional sequence in $J(M) \cong \bmod \Gamma_{n-1}^{2}$. Hence the number of complete $\tau$-exceptional sequences ending in $M$ is $G_{n-1}$, which is the number of complete $\tau$-exceptional sequences in $\bmod \Gamma_{n-1}^{2}$.

Now suppose $M$ is a simple $\Lambda_{n}^{2}$-module. By Lemma 5.2, the $\tau$-perpendicular category $J(M) \cong \bmod \Gamma_{n-2}^{2} \oplus \bmod \Gamma_{1}^{2}$. Arguing as above the number of complete $\tau$-exceptional sequences ending in $M$ is equal to the number of complete $\tau$-exceptional sequences in $J(M)$. Since $J(M) \cong \bmod \Gamma_{n-2}^{2} \oplus \bmod \Gamma_{1}^{2}$, by Theorem 3.8 and 3.9, the $\tau$-exceptional sequences of $J(M)$ are interleavings of $\tau$-exceptional sequences of $\bmod \Gamma_{n-2}^{2}$ and $\bmod \Gamma_{1}^{2}$. The number of interleaved sequences coming from a sequence of length $n-2$ and a sequence of length 1 is precisely $\left(\begin{array}{c}n-1 \\ n-2,1\end{array}\right)=(n-1)$. Thus the number of complete $\tau$-exceptional sequences ending in $M$ is $(n-1) G_{n-2} G_{1}=(n-1) G_{n-2}$.

An arbitrary indecomposable $\Lambda_{n}^{2}$-module is either projective or simple. There are $n$ projective modules and $n$ simple modules up to isomorphism in $\bmod \Lambda_{n}^{2}$, hence the number of complete $\tau$-exceptional sequences in $\bmod \Lambda_{n}^{2}$ is $L_{n}=n G_{n-1}+n(n-1) G_{n-2}$. It then follows easily that $L_{1}=1$ and $L_{2}=4$.

In the previous section we found the exponential generating function and closed formula for $G_{n}$. Using the above theorem, we can immediately do the same for $L_{n}$.

Theorem 5.4 Let $L_{n}$ denote the number of complete $\tau$-exceptional sequences in mod $\Lambda_{n}^{2}$. The exponential generating function of $L_{n}$ is as follows,

$$
\sum_{n=0}^{\infty} L_{n} \frac{x^{n}}{n !}=\frac{x+x^{2}}{1-x-\frac{x^{2}}{2}}
$$

Proof Let $h(x)=\sum_{n=0}^{\infty} L_{n} \frac{x^{n}}{n !}$ be the exponential generating function of $L_{n}$. Let

$$
g(x)=\sum_{n=0}^{\infty} G_{n} \frac{x^{n}}{n !}
$$

be the exponential generating function of $G_{n}$. We then recall the recurrence relation of $L_{n}$,

$$
L_{n}=n G_{n-1}+n(n-1) G_{n-2} .
$$

Therefore the exponential generating function of $L_{n}$ is,

$$
\begin{gathered}
\sum_{n=0}^{\infty} L_{n} \frac{x^{n}}{n !}=\sum_{n=0}^{\infty} n G_{n-1} \frac{x^{n}}{n !}+\sum_{n=0}^{\infty} n(n-1) G_{n-2} \frac{x^{n}}{n !} \\
=\sum_{n=0}^{\infty} G_{n-1} \frac{x^{n}}{(n-1) !}+\sum_{n=0}^{\infty} G_{n-2} \frac{x^{n}}{(n-2) !}
\end{gathered}
$$




$$
=x \sum_{n=0}^{\infty} G_{n-1} \frac{x^{n-1}}{(n-1) !}+x^{2} \sum_{n=0}^{\infty} G_{n-2} \frac{x^{n-2}}{(n-2) !} .
$$

Therefore

$$
h(x)=x g(x)+x^{2}(g(x))=\left(x+x^{2}\right) g(x) .
$$

By Theorem 4.6,

$$
g(x)=\frac{1}{1-x-\frac{x^{2}}{2}},
$$

hence

$$
h(x)=\frac{x+x^{2}}{1-x-\frac{x^{2}}{2 !}} .
$$

Theorem 5.5 Let $L_{n}$ denote the number of complete $\tau$-exceptional sequences in $\bmod \Lambda_{n}^{2}$. Then $L_{n}$ is given by the closed formula,

$$
L_{n}=\frac{n !}{\sqrt{3}}\left((\sqrt{3}-1)^{-n-2}-(-\sqrt{3}-1)^{-n-2}\right)+\frac{n !}{\sqrt{3}}\left((\sqrt{3}-1)^{-n-3}-(-\sqrt{3}-1)^{-n-3}\right) .
$$

Proof It is immediate from the recurrence relation for $L_{n}$ and Theorem 4.6 that,

$$
\begin{gathered}
L_{n}=n \frac{(n-1) !}{\sqrt{3}}\left((\sqrt{3}-1)^{-n-2}-(-\sqrt{3}-1)^{-n-2}\right)+n(n-1) \frac{(n-2) !}{\sqrt{3}}\left((\sqrt{3}-1)^{-n-3}-(-\sqrt{3}-1)^{-n-3}\right) \\
=\frac{n !}{\sqrt{3}}\left((\sqrt{3}-1)^{-n-2}-(-\sqrt{3}-1)^{-n-2}\right)+\frac{n !}{\sqrt{3}}\left((\sqrt{3}-1)^{-n-3}-(-\sqrt{3}-1)^{-n-3}\right) .
\end{gathered}
$$

We calculate the first 10 terms of the sequence $\left(L_{n}\right)_{n=0}^{\infty}$ to be,

$$
1,4,15,84,570,4680,44730,488880,6010200,82101600 .
$$

In comparison to $\tau$-exceptional sequences, there are no complete exceptional sequences in $\bmod \Lambda_{n}^{2}$, as we will show. In general, not much is known about exceptional sequences over the Nakayama algebras $\Lambda_{n}^{2}$.

Proposition 5.6 There are no complete exceptional sequences in $\bmod \Lambda_{n}^{2}$ when $n>1$.

Proof Suppose $M=\left(M_{1}, M_{2}, \ldots, M_{n}\right)$ is a complete exceptional sequence. Recall that an indecomposable module in $\bmod \Lambda_{n}^{2}$ is either projective or simple. Since $\operatorname{Hom}\left(P_{i}, P_{(i+1)_{n}}\right) \neq 0$ for $1 \leq i \leq n$, the sequence $M$ cannot consist entirely of just indecomposable projective modules, so $M$ must contain at least one simple module.

Consider the simple module $S_{i}$ for some $1 \leq i \leq n$. Then $S_{i}$ has the following infinite exact sequence as its projective resolution.

$$
\ldots \longrightarrow P_{1} \longrightarrow P_{n} \longrightarrow \ldots \longrightarrow P_{1} \longrightarrow P_{n} \longrightarrow \ldots \rightarrow P_{i+1} \longrightarrow P_{i} \longrightarrow S_{i} \longrightarrow 0
$$

We observe that the projective resolution of $S_{i}$ contains every projective indecomposable module of $\bmod \Lambda_{n}^{2}$. We also observe that the only projective module $P$ such that $\operatorname{Hom}\left(P, S_{i}\right) \neq 0$ is $P=P_{i}$. Hence applying the functor $\operatorname{Hom}\left(-, S_{i}\right)$ to the above projective resolution we get the following sequence.

$$
0 \longrightarrow \operatorname{Hom}\left(S_{i}, S_{i}\right) \stackrel{f_{0}}{\longrightarrow} \operatorname{Hom}\left(P_{i}, S_{i}\right) \stackrel{f_{1}}{\longrightarrow} \ldots \stackrel{f_{n-1}}{\longrightarrow} 0 \stackrel{f_{n}}{\longrightarrow} \operatorname{Hom}\left(P_{i}, S_{i}\right) \stackrel{f_{n+1}}{\longrightarrow} 0 \stackrel{f_{n+2}}{\longrightarrow} \ldots
$$


We then observe that $\operatorname{Ext}^{n}\left(S_{i}, S_{i}\right)=\operatorname{ker}\left(f_{n+1}\right) / \operatorname{im}\left(f_{n}\right) \neq 0$, so no simple module in $\bmod \Lambda_{n}^{2}$ is exceptional. From this we conclude $M$ cannot contain simple modules, a contradiction.

Unlike exceptional sequences, weak exceptional sequences number over $\Lambda_{n}^{2}$ have been studied. Weak exceptional sequences are defined in [30] as follows.

Definition 5.7 [30, Definition 1.1]. Let $A$ be a finite dimensional algebra over a field $\mathbb{F}$, where $\mathbb{F}$ is algebraically closed. A left $A$-module $M$ is called weak exceptional if $\operatorname{Hom}(M, M) \cong \mathbb{F}$ and $\operatorname{Ext}_{A}^{1}(M, M)=0$. A sequence of indecomposable modules $\left(M_{1}, M_{2}, \ldots, M_{r}\right)$ is called a weak exceptional sequence if, for each pair $\left(M_{i}, M_{j}\right)$ with $1 \leq i<j \leq r$, we have that $\operatorname{Hom}\left(M_{j}, M_{i}\right)=\operatorname{Ext}_{A}^{1}\left(M_{j}, M_{i}\right)=0$ and each $M_{k}$ is weak exceptional for $1 \leq k \leq r$.

It turns out that for weak exceptional sequences over $\Lambda_{n}^{2}$, the maximum length need not be $n$ and in fact can exceed $n$. According to [30, Theorem 1.6], if $n=2 m+1$ is odd, the maximum length of a weak exceptional sequence over $\Lambda_{n}^{2}$ is equal to $3 m+1$. On the other hand, if $n=2 m$ is even, then the maximum length of a weak exceptional sequence over $\Lambda_{n}^{2}$ is $3 m-1$. A weak exceptional sequence with maximum length is called full. Again by [30, Theorem 1.6], when $n=2 m$, the number of full weak exceptional sequences is given by $2 m\left(\frac{8^{m}}{12}-\frac{(-1)^{m}}{3}+1\right)$, and when $n=2 m+1$, the number of full weak exceptional sequences is given by $n$.

\section{The $\Lambda_{n}^{n}$ Case}

Let $n \geq 1$ be a positive integer. In this section we will derive a closed formula for the number of complete $\tau$-exceptional sequences in $\bmod \Lambda_{n}^{n}$. Recall that we denote by $C_{n}$ the linearly oriented $n$-cycle.

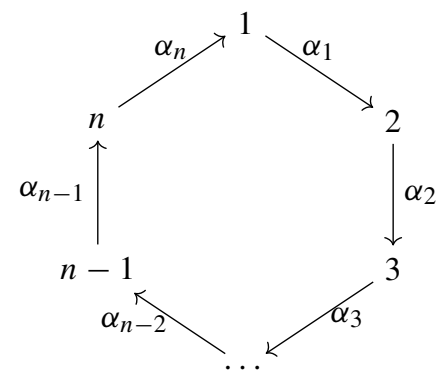

The algebra $\Lambda_{n}^{n}$ is defined to be the $\mathbb{F}$-algebra, $\mathbb{F} C_{n} / R_{Q}^{n}$. This is the path algebra of the quiver $C_{n}$ modulo the relations $\alpha_{i} \alpha_{(i+1)_{n}} \ldots \alpha_{(i+(n-1))_{n}}=0$ for $1 \leq j \leq n$.

Proposition 6.1 [1, Proposition 2.5]. Let $A$ be a Nakayama algebra. Let $M$ be an indecomposable non-projective module in $\bmod A$. Then $M$ is rigid if and only if $l(M)<n$ holds.

For our purposes, the following Proposition is a more convenient restatement of Proposition 3.12. 
Proposition 6.2 Let $M$ be an indecomposable $\Lambda_{n}^{n}$-module with length $1 \leq l(M) \leq n-1$. Then $\operatorname{Hom}(X, \tau M) \neq 0$ if and only if top $(X) \cong \operatorname{top}\left(\operatorname{rad}^{k}(\tau M)\right)$ for some $0 \leq k \leq l(M)-1$ and $l\left(\operatorname{rad}^{k}(\tau M)\right) \leq l(X)$.

Proof All indecomposable modules in $\bmod \Lambda_{n}^{n}$ have simple tops. By Proposition 3.11, for a $\Lambda_{n}^{n}$-module $X$, we have that $X=P_{j} / \operatorname{rad}^{l(X)}\left(P_{j}\right)$ hence $\operatorname{top}(X)=S_{j}$. Let $M=$ $P_{i-1} / \mathrm{rad}^{l(M)} P_{i-1}$, then $\tau M=P_{(i)_{n}} / \mathrm{rad}^{l(M)}\left(P_{(i)_{n}}\right)$ by Proposition 3.11 as well. Observe that for $0 \leq k \leq l(M)-1, \operatorname{rad}^{k}(\tau M)=P_{(i+k)_{n}} / \operatorname{rad}^{(l(M)-k)}\left(P_{(i+k)_{n}}\right)$ thus top $\left(\operatorname{rad}^{k}(\tau M)\right)=$ $S_{(i+k)_{n}}$ and $l\left(\operatorname{rad}^{k}(\tau M)\right)=l(M)-k$. By Proposition 3.12 we have that,

$\operatorname{Hom}(X, \tau M) \neq 0$ if and only if $j \in[i,(i+l(M)-1)]_{n}$ and $(i+l(M)-1)_{n} \in[j,(j+l(X)-1)]_{n}$.

Suppose $\operatorname{Hom}(X, \tau M) \neq 0$, this implies that $j=(i+k)_{n}$ for some $0 \leq k \leq l(M)-1$, and $(i+l(M)-1)_{n}=(j+a)_{n}$ for some $0 \leq a \leq l(X)-1$. It then immediately follows $\operatorname{top}(X) \cong \operatorname{top}\left(\operatorname{rad}^{k}(\tau M)\right)$ and $l\left(\operatorname{rad}^{k}(\tau M)\right) \leq l(X)$.

For the converse, suppose that $\operatorname{top}(X) \cong \operatorname{top}\left(\operatorname{rad}^{k}(\tau M)\right)$ and $l\left(\operatorname{rad}^{k}(\tau M)\right) \leq l(X)$. Then $j=(i+k)_{n}$ for some $0 \leq k \leq l(M)-1$. Moreover, $l\left(\operatorname{rad}^{k}(\tau M)\right)=l(M)-k \leq l(X)$ which implies $i+l(M)-1 \leq(i+k)+l(X)-1$ therefore $(i+l(M)-1)_{n} \in[j,(j+l(X)-1)]_{n}$. Hence by Proposition 3.12, $\operatorname{Hom}(X, \tau M) \neq 0$.

By Proposition 6.1, every indecomposable module $M$ of $\bmod \Lambda_{n}^{n}$ is $\tau$-rigid in $\bmod \Lambda_{n}^{n}$ since it is either projective or has length $l(M)<n$. Hence, we once again adopt the same strategy for calculating the number of complete $\tau$-exceptional sequences in $\bmod \Lambda_{n}^{n}$ as we have done thus far. For each $M$ in ind $\left(\bmod \Lambda_{n}^{n}\right)$, we will calculate the number of complete $\tau$-exceptional sequences ending in $M$. By definition a sequence of indecomposable modules $\left(M_{1}, M_{2}, \ldots, M_{n-1}, M\right)$ is a $\tau$-exceptional sequence in $\bmod \Lambda_{n}^{n}$ if $M$ is $\tau$-rigid and $\left(M_{1}, M_{2}, \ldots, M_{n-1}\right)$ is a $\tau$-exceptional sequence in $J(M)$. Having seen that every indecomposable $\Lambda_{n}^{n}$-module $M$ is $\tau$-rigid, what is left to do is to calculate $J(M)$ for each indecomposable module. Theorem 3.5 and Lemma 3.4 are once again the main tools these calculations.

Proposition 6.3 Let $M$ be an indecomposable $\Lambda_{n}^{n}$-module with length $1 \leq l(M) \leq n-1$ and $\operatorname{top}(M)=S_{i}$. Then for all $1 \leq k \leq l(M)-1$,

$$
\mathcal{P}\left({ }^{\perp}(\tau M)\right)=M \oplus \bigoplus_{s=1}^{l(M)-1} \operatorname{rad}^{s}(M) \oplus \bigoplus_{\substack{1 \leq j \leq n \\ j \notin[i+1, i+l(M)]_{n}}} P_{j}
$$

Proof Suppose the $\Lambda_{n}^{n}$-module $M$ has top equal to top $(M)=S_{i}$ and has length $1 \leq$ $l(M) \leq n-1$ i.e. $M$ is not projective. By Proposition 3.11, $M=P_{i} / \mathrm{rad}^{l(M)}\left(P_{i}\right)$ and $\tau M=\operatorname{rad}\left(P_{i}\right) / \operatorname{rad}^{l(M)+1}\left(P_{i}\right)$ with $l(M)=l(\tau M)$. It is easy to see that $\operatorname{top}(\tau M)=S_{(i+1)_{n}}$ hence $\tau M=P_{(i+1)_{n}} / \operatorname{rad}^{l(M)}\left(P_{(i+1)_{n}}\right)$.

By Proposition 6.2, a $\Lambda_{n}^{n}$-module $X$ is not in ${ }^{\perp}(\tau M)$ if and only if $\operatorname{top}(X) \cong$ top $\left(\operatorname{rad}^{k}(\tau M)\right)$ for some $0 \leq k \leq l(M)-1$ and $l\left(\operatorname{rad}^{k}(\tau M)\right) \leq l(X)$. Let $X=$ $P_{j} / \operatorname{rad}^{l(X)}\left(P_{j}\right)$ for some $1 \leq j \leq n$. The statement $\operatorname{top}(X) \cong \operatorname{top}\left(\operatorname{rad}^{k}(\tau M)\right)$ for some $0 \leq k \leq l(M)-1$ means that $j=(i+1+k)_{n}$ for some $0 \leq k \leq l(M)-1$. With this we are able to determine the Ext-projectives in ${ }^{\perp}(\tau M)$.

Let $Y=P_{l}$ be the indecomposable project at the vertex $l$ with $l \neq(i+1+k)_{n}$ for some $0 \leq k \leq l(M)-1$. Then $P_{l}$ is in ${ }^{\perp}(\tau M)$ by Proposition 6.2. Moreover $\operatorname{Ext}_{\Lambda_{n}^{n}}\left(P_{l},{ }^{\perp}(\tau M)\right)=$ 0 since $P_{l}$ is a projective $\Lambda_{n}^{n}$-module. Hence $P_{l}$ is Ext-projective in ${ }^{\perp}(\tau M)$. 
Let $Y=\operatorname{rad}^{s}(M)$ for some $1 \leq s \leq l(M)-1$. Then observe that $Y=$ $P_{(i+s)_{n}} / \operatorname{rad}^{(l(M)-s)}\left(P_{(i+s)_{n}}\right)$ meaning $l(\bar{Y})=l(M)-s$. Recall a $\Lambda_{n}^{n}$-module $X$ is not in $\perp(\tau Y)$ if and only if top $(X) \cong \operatorname{top}\left(\operatorname{rad}^{r}(\tau Y)\right)$ for some $0 \leq r \leq l(M)-s-1$ and $l\left(\operatorname{rad}^{r}(\tau Y)\right) \leq l(X)$. Therefore if $X=P_{j} / \operatorname{rad}^{l(X)}\left(P_{j}\right)$, then $j=(i+1+s+r)_{n}$ for some $0 \leq r \leq l(M)-s-1$. This implies that $\{X: \operatorname{Hom}(X, \tau Y) \neq 0\} \subset\{X: \operatorname{Hom}(X, \tau M) \neq 0\}$, which further implies that $\operatorname{Ext}_{\Lambda_{n}^{n}}(Y, N) \cong \overline{\mathrm{DHom}}_{\Lambda_{n}^{n}}(N, \tau Y)=0$ for all $N$ in ${ }^{\perp}(\tau M)$ by the Auslander-Reiten formula. Hence $Y=\operatorname{rad}^{S}(M)$ is an Ext-projective in ${ }^{\perp}(\tau M)$.

By Proposition 3.1, $M$ is Ext-projective in ${ }^{\perp}(\tau M)$. For every other indecomposable $\Lambda_{n}^{n}$ module $Y$, we have that $\tau Y$ is in ${ }^{\perp}(\tau M)$, therefore $\operatorname{Ext}_{\Lambda_{n}^{n}}(Y, \tau Y) \cong \overline{\mathrm{Dom}}_{\Lambda_{n}^{n}}(\tau Y, \tau Y) \neq 0$ i.e. they are not Ext-projective in ${ }^{\perp}(\tau M)$. By definition, $T_{M}=\mathcal{P}\left({ }^{\perp}(\tau M)\right)$, hence by the above arguments,

$$
\mathcal{P}\left({ }^{\perp}(\tau M)\right)=M \oplus \bigoplus_{s=1}^{l(M)-1} \operatorname{rad}^{s}(M) \oplus \bigoplus_{\substack{1 \leq j \leq n \\ j \notin[i+1, i+l(M)]_{n}}} P_{j} .
$$

Proposition 6.4 Let $P_{i}$ be an indecomposable projective module in $\bmod \Lambda_{n}^{n}$ for some $1 \leq$ $i \leq n$. Then the $\tau$-perpendicular category of $P_{i}$ in $\bmod \Lambda_{n}^{n}$ is $J\left(P_{i}\right) \cong \bmod \mathbb{A}_{n-1}$, where $\mathbb{A}_{n-1}$ is the Dynkin type A hereditary algebra.

Proof By definition $T_{P_{i}}=\mathcal{P}\left({ }^{\perp}\left(\tau P_{i}\right)\right)$. Since $P_{i}$ is projective, we have that $\tau P_{i}=0$ therefore ${ }^{\perp}\left(\tau P_{i}\right)=\bmod \Lambda_{n}^{n}$. As a result the Ext-projectives of ${ }^{\perp}\left(\tau P_{i}\right)$ are just the projectives of $\bmod \Lambda_{n}^{n}$ therefore

$$
T_{P_{i}}=\mathcal{P}\left({ }^{\perp}\left(\tau P_{i}\right)\right)=\bigoplus_{j=1}^{n} P_{j} .
$$

Thus the $\mathbb{F}$-algebra $E_{P_{i}}=\operatorname{End}_{\Lambda_{n}^{2}}\left(T_{P_{i}}\right)$ is precisely given by the path algebra of the quiver $C_{n}^{\mathrm{op}}$,

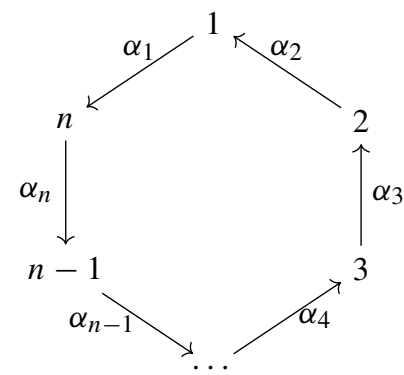

modulo the relations $\alpha_{i} \alpha_{(i+1)_{n}} \ldots \alpha_{(i+(n-1))_{n}}=0$ for $1 \leq j \leq n$.

By Lemma 3.4, $D_{M}=E_{M} /\left\langle e_{M}\right\rangle$ is the path algebra of the quiver $C_{n}^{\text {op }(i)}$ which is the quiver obtained from $C_{n}^{\text {op }}$ by removing the vertex $i$. More precisely, $C_{n}^{\mathrm{op}(i)}$ is the quiver,

$$
i+1 \stackrel{\alpha_{i+2}}{\longleftarrow} i+2 \stackrel{\alpha_{i+3}}{\longleftarrow} \ldots \stackrel{\alpha_{n-1}}{\longleftarrow} n-1 \stackrel{\alpha_{n}}{\longleftarrow} n \stackrel{\alpha_{1}}{\longleftarrow} 1 \stackrel{\alpha_{2}}{\longleftarrow} \ldots \stackrel{\alpha_{i-2}}{\longleftarrow} i-2 \stackrel{\alpha_{i-1}}{\longleftarrow} i-1
$$

with no relations. It is easy to see that the path algebra $\mathbb{F} C_{n}^{\text {op }(i)}$ is isomorphic to $\mathbb{A}_{n-1}$. Hence the Proposition follows by Theorem 3.5.

Proposition 6.5 Let $M$ be an indecomposable $\Lambda_{n}^{n}$-module with length $1 \leq l(M) \leq n-1$ and $\operatorname{top}(M)=S_{i}$. Which is to say that $M=P_{i} / \operatorname{rad}^{l(M)}\left(P_{i}\right)$. Then the $\tau$-perpendicular 
category of $M$ in $\bmod \Lambda_{n}^{n}$ is $J(M) \cong \bmod \mathbb{A}_{l(M)-1} \oplus \bmod \Lambda_{n-l(M)}^{n-l(M)}$, where $\mathbb{A}_{m}$ is the Dynkin type A hereditary algebra.

Proof By Proposition 6.3 the Bongartz completion of $M$ is,

$$
T_{M}=M \oplus \bigoplus_{s=1}^{l(M)-1} \operatorname{rad}^{s}(M) \oplus \bigoplus_{\substack{1 \leq j \leq n \\ j \notin[i+1, i+l(M)]_{n}}} P_{j}
$$

Hence the $\mathbb{F}$-algebra $E_{M}=\operatorname{End}_{\Lambda_{n}^{n}}\left(T_{M}\right)$ is the path algebra of the following quiver $Q_{n}$,

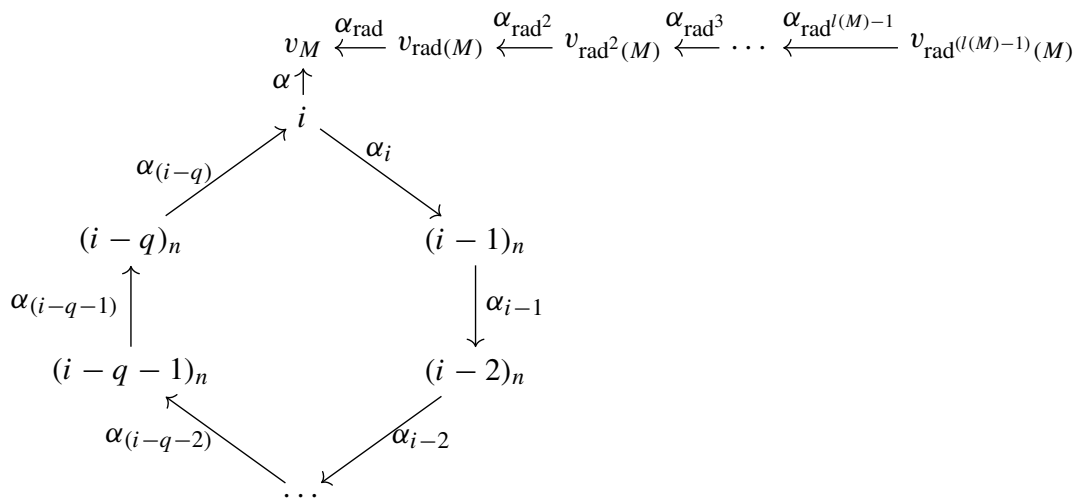

modulo the relations $\alpha_{r} \alpha_{r-1} \ldots \alpha_{r-(n-l(M)-1)}=0$ for $r \in[i, i-(n-l(M)-1)]_{n}$ and where $q=n-l(M)-1$

Let $Q_{n}^{\left(v_{M}\right)}$ be the quiver obtained from $Q_{n}$ by removing the vertex $v_{M}$ and any arrows incident to $v_{M}$. More precisely, $Q_{n}^{\left(v_{M}\right)}$ is the following quiver with two connected components,

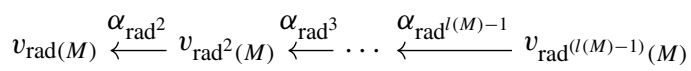

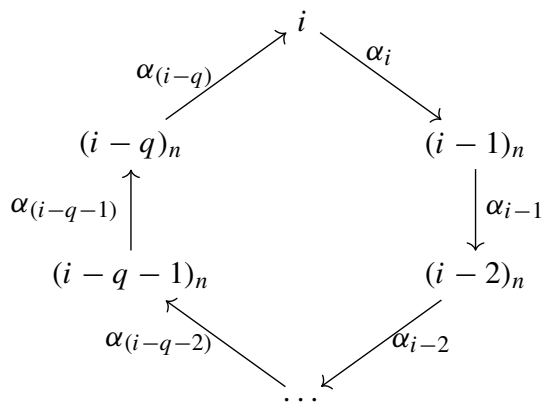

and with relations $\alpha_{r} \alpha_{r-1} \ldots \alpha_{r-(n-l(M)-1)}=0$ for $r \in[i, i-(n-l(M)-1)]_{n}$. By Lemma 3.4, $D_{M}=E_{M} /\left\langle e_{M}\right\rangle$ is the path algebra of $Q_{n}^{\left(v_{M}\right)}$ modulo relations. So it follows that $\bmod D_{M} \cong$ $\bmod \mathbb{A}_{l(M)-1} \oplus \bmod \Lambda_{n-l(M)}^{n-l(M)}$. So by Theorem 3.5, the statement of the Proposition follows. 
Theorem 6.6 Let $H_{n}$ denote the number of complete $\tau$-exceptional sequences in $\bmod \Lambda_{n}^{n}$. Then $H_{n}$ satisfies the recurrence relation,

$$
H_{n}=n \sum_{i=1}^{n}\left(\begin{array}{c}
n-1 \\
i-1
\end{array}\right) i^{i-2} H_{n-i}
$$

with $H_{0}=1$.

Proof Let $M$ be an indecomposable $\Lambda_{n}^{n}$-module. Suppose $\left(M_{1}, M_{2}, \ldots, M_{n-1}, M\right)$ is a complete $\tau$-exceptional sequence in $\bmod \Lambda_{n}^{n}$ ending in $M$. Then by definition and the fact that $\delta(J(M))=n-1$, the sequence $\left(M_{1}, M_{2}, \ldots, M_{n-1}\right)$ is a complete $\tau$-exceptional sequence in $J(M)$. It then follows that the number of complete $\tau$-exceptional sequences ending in $M$ is equal to the number of complete $\tau$-exceptional sequences in $J(M)$.

The length of $M$ is $1 \leq l(M) \leq n$. For each possible value of $l(M)$, there are $n$ indecomposable $\Lambda_{n}^{n}$-modules of that length. If $l(M)=n$ then $M$ is projective and by Proposition $6.4, J(M) \cong \bmod \mathbb{A}_{n-1}$. The number of $\tau$-exceptional sequences in $\bmod \mathbb{A}_{n-1}$ was shown in [[28] [Proposition 1.1]] to be $n^{n-2}=\left(\begin{array}{c}n-1 \\ n-1\end{array}\right) n^{n-2} H_{0}$, where $H_{0}=1$.

If $1 \leq l(M) \leq n-1$, then by Proposition 6.5, the $\tau$-perpendicular category of $M$ is $J(M) \cong \bmod \mathbb{A}_{l(M)-1} \oplus \bmod \Lambda_{n-l(M)}^{n-l(M)}$. Arguing as above, the number of complete $\tau$-exceptional sequences ending in $M$ is equal to the number of complete $\tau$-exceptional sequences in $\bmod \mathbb{A}_{l(M)-1} \oplus \bmod \Lambda_{n-l(M)}^{n-l(M)}$. By Theorems 3.8 and 3.9, this is equal to

$$
\left(\begin{array}{c}
n-1 \\
n-l(M), l(M)-1
\end{array}\right) l(M)^{(l(M)-2)} H_{n-l(M)}=\left(\begin{array}{c}
n-1 \\
l(M)-1
\end{array}\right) l(M)^{(l(M)-2)} H_{n-l(M)} .
$$

So it follows that,

$$
H_{n}=\sum_{l(M)=1}^{n} n\left(\begin{array}{c}
n-1 \\
l(M)-1
\end{array}\right) l(M)^{l(M)-2} H_{n-l(M)}=n \sum_{i=1}^{n}\left(\begin{array}{c}
n-1 \\
i-1
\end{array}\right) i^{i-2} H_{n-i} .
$$

It is trivial to see that $H_{1}=1$. Using the recurrence we obtain $H_{1}=\left(\begin{array}{l}0 \\ 0\end{array}\right) 1^{-1} H_{0}=1$, therefore $H_{0}=1$.

We are now in a position to derive the exponential generating function of $H_{n}$. First we state the following results and definitions which will be useful in deriving the exponential generating function.

Lemma 6.7 [31, Section 2.3 Rule 3']. Let $f=\sum_{n=0}^{\infty} a_{n} \frac{x^{n}}{n !}$ and $g=\sum_{n=0}^{\infty} b_{n} \frac{x^{n}}{n !}$ be the generating functions of the sequences $\left\{a_{n}\right\}_{n=0}^{\infty}$ and $\left\{b_{n}\right\}_{n=0}^{\infty}$ respectively. Then the series $f g$ is the exponential generating function of the sequence,

$$
\left\{\sum_{k=0}^{n}\left(\begin{array}{l}
n \\
k
\end{array}\right) a_{k} b_{n-k}\right\}_{n=0}^{\infty}
$$

The Lambert $W$ function is defined to be the function $W(z)$ satisfying $W(z) e^{W(z)}=z$. The tree function $T(z)$ is defined by the equation $T(z)=-W(-z)$. The functions $W$ and $T$ have many applications in mathematics. For example, they appear in the enumeration of trees and the calculation of water-wave heights. The reader is referred to [9] for more on Lambert's $W$ function. 
Lemma 6.8 [9, Section 2, Equation 2.36]. Let $a \geq 1$ and $n \geq 0$ be integers. Let $N(a, n):=$ $a(a+n)^{n-1}$ be a function of two variables. For a fixed positive integer $a$, the exponential generating function of the sequence $N(a, n)$ is given by,

$$
\sum_{n=0}^{\infty} a(a+n)^{n-1} \frac{x^{n}}{n !}=e^{-a W(-x)},
$$

where $W$ is Lambert's $W$ function.

Theorem 6.9 The exponential generating function of $H_{n}$ is,

$$
\sum_{n=0}^{\infty} H_{n} \frac{x^{n}}{n !}=\frac{1}{1+W(-x)}
$$

where $W$ is Lambert's $W$ function and $H_{n}$ is given by the closed formula,

$$
H_{n}=n^{n} \text {. }
$$

Proof Let $a_{n}$ be the sequence $a_{n}=(n+1)^{n-1}$. Let $h(x)=\sum_{n=0}^{\infty} H_{n} \frac{x^{n}}{n !}$ and $g(x)=$ $\sum_{n=0}^{\infty} a_{n} \frac{x^{n}}{n !}$ be exponential generating functions of $H_{n}$ and $a_{n}$ respectively. Recall the recurrence relation of $H_{n}$ is given by,

$$
H_{n}=n \sum_{k=1}^{n}\left(\begin{array}{l}
n-1 \\
k-1
\end{array}\right) k^{k-2} H_{n-k}
$$

so,

$$
\frac{H_{n}}{n}=\sum_{k=1}^{n}\left(\begin{array}{l}
n-1 \\
k-1
\end{array}\right) k^{k-2} H_{n-k} .
$$

We make the change of variable $j=k-1$ in $\frac{H_{n}}{n}$ to obtain the following.

$$
\frac{H_{n}}{n}=\sum_{j=0}^{n-1}\left(\begin{array}{c}
n-1 \\
j
\end{array}\right)(j+1)^{j-1} H_{n-(j+1)},
$$

thus

$$
\frac{H_{n+1}}{n+1}=\sum_{j=0}^{n}\left(\begin{array}{l}
n \\
j
\end{array}\right)(j+1)^{j-1} H_{n-j} .
$$

We now study the exponential generating function of $\frac{H_{n+1}}{n+1}$,

$$
\sum_{n=0}^{\infty} \frac{H_{n+1}}{n+1} \frac{x^{n}}{n !}=\sum_{n=0}^{\infty}\left(\sum_{j=0}^{n}\left(\begin{array}{c}
n \\
j
\end{array}\right)(j+1)^{j-1} H_{n-j}\right) \frac{x^{n}}{n !} .
$$

By Lemma 6.7, the right hand side is given the product $g(x) h(x)$. So we have,

$$
\sum_{n=0}^{\infty} \frac{H_{n+1}}{n+1} \frac{x^{n}}{n !}=g(x) h(x) .
$$

We can manipulate the right hand side so that the exponent of $x$ matches the factorial, hence

$$
\frac{1}{x} \sum_{n=0}^{\infty} H_{n+1} \frac{x^{n+1}}{(n+1) !}=g(x) h(x),
$$


so we can write the left hand side in terms of $h(x)$ as follows,

$$
\frac{1}{x}\left(h(x)-H_{0}\right)=g(x) h(x) .
$$

Since $H_{0}=1$,

$$
h(x)-1=x h(x) g(x) .
$$

By Lemma 6.8, $g(x)=e^{-W(-x)}$ therefore,

$$
h(x)=\frac{1}{1-x g(x)}=\frac{1}{1-x e^{-W(-x)}} .
$$

Recall that Lambert's $W$ function is defined by the equation $x=W(x) e^{W(x)}$ (See [9] for more on Lambert's $W$ function), thus $-x e^{-W(-x)}=W(-x)$, giving us that,

$$
h(x)=\frac{1}{1+W(-x)}=\frac{1}{1-T(x)},
$$

where $T(x)=-W(-x)$ is called Euler's tree function, again see [9]. This exponential generating function is precisely the exponential generating function of the sequence $n^{n}$, see; [19] Section 2 equation 2.7 and [26].

It is interesting to note that $n^{n}$ is also the number of complete exceptional sequences over the hereditary algebras of type B and C; see section 5 of [22]. On a more interesting note, $n^{n}$ also counts the number of full weak exceptional sequences (see Definition 5.7) over $\Lambda_{n}^{n}$ [30, Theorem 1.4]. The full weak exceptional sequences over $\Lambda_{n}^{n}$ also have length $n$, so a natural question to ask is whether the complete $\tau$-exceptional sequences over $\Lambda_{n}^{n}$ coincide with the full weak exceptional sequences (see Definition 5.7) over $\Lambda_{n}^{n}$. We answer this question in the affirmative. First, we state the following well known result.

Lemma 6.10 [8, Lemma 10.20] Let $\mathcal{A}$ be an exact category and let $\mathcal{B}$ be a full additive subcategory of $\mathcal{A}$. Then if $\mathcal{B}$ is extension-closed in $\mathcal{A}$, the exact sequences $A \rightarrow B \rightarrow C$ in $\mathcal{A}$ with $A, B$, and $C \in \mathcal{B}$ form an exact structure on $\mathcal{B}$. In particular for $X, Y \in \mathcal{B}$, we have that $\operatorname{Ext}_{\mathcal{A}}^{1}(X, Y)=\operatorname{Ext}_{\mathcal{B}}^{1}(X, Y)$.

Lemma 6.11 [18, Proposition 3.6] Let $A$ be a finite dimensional $\mathbb{F}$ algebra and let $M$ be a basic $\tau$-rigid left A-module. Then the $\tau$-perpendicular category $J(M)$ is extension-closed in $\bmod A$.

Proposition 6.12 Let $M=\left(M_{1}, M_{2}, \ldots, M_{n}\right)$ be a complete $\tau$-exceptional sequence in $\bmod \Lambda_{n}^{n}$. Then $M$ is also a full weak exceptional sequence in $\bmod \Lambda_{n}^{n}$.

Proof We will argue by induction on $n$. In the case of $n=1$, there is only one indecomposable module which is both weak exceptional and $\tau$-rigid, so the statement follows trivially. Suppose that the statement is true for all $1 \leq n \leq k$. Let us consider the $k+1$ case. Suppose $M=\left(M_{1}, M_{2}, \ldots, M_{k+1}\right)$ is a $\tau$-exceptional sequence in $\bmod \Lambda_{k+1}^{k+1}$.

Let $l=l\left(M_{k+1}\right)$ be the length of $M_{k+1}$, then $1 \leq l \leq k+1$. By Propositions 6.4 and 6.5 , the $\tau$-perpendicular category

$$
J\left(M_{k+1}\right) \cong \bmod \mathbb{A}_{l-1} \oplus \Lambda_{k+1-l}^{k+1-l} .
$$

By definition, the sequence $\left(M_{1}, M_{2}, \ldots, M_{k}\right)$ is $\tau$-exceptional in $J\left(M_{k+1}\right)$. By Theorems 3.8 and 3.9, we have that the sequence $\left(M_{1}, M_{2}, \ldots, M_{k}\right)$ is an interleaving of a complete exceptional sequence $X=\left(X_{1}, X_{2}, \ldots, X_{l-1}\right)$ in $\bmod \mathbb{A}_{l-1}$ with a complete 
$\tau$-exceptional sequence $Y=\left(Y_{1}, Y_{2}, \ldots, Y_{k+1-l}\right)$ in $\bmod \Lambda_{k+1-l}^{k+1-l}$. By the inductive hypothesis, the sequence $Y$ is a full weak exceptional sequence in $\bmod \Lambda_{k+1-l}^{k+1-l}$. Moreover, we have that

$$
\operatorname{Hom}_{J\left(M_{k+1}\right)}\left(X_{i}, Y_{j}\right)=0=\operatorname{Hom}_{J\left(M_{k+1}\right)}\left(Y_{j}, X_{i}\right) \text {, }
$$

where $1 \leq i \leq l-1$ and $1 \leq j \leq k+1-l$. Therefore, since the $\tau$-perpendicular category $J\left(M_{k+1}\right)$ is a full subcategory of $\bmod \Lambda_{k+1}^{k+1}$, we also have that

$$
\operatorname{Hom}_{\bmod \Lambda_{k+1}^{k+1}}\left(X_{i}, Y_{j}\right)=0=\operatorname{Hom}_{\bmod \Lambda_{k+1}^{k+1}}\left(Y_{j}, X_{i}\right),
$$

where $1 \leq i \leq l-1$ and $1 \leq j \leq k+1-l$. By a similar argument, we also have that

$$
\operatorname{Hom}_{\bmod \Lambda_{k+1}^{k+1}}\left(X_{j}, X_{i}\right)=0
$$

for $1 \leq i<j \leq l-1$ and

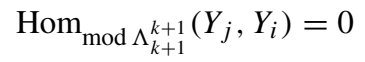

for $1 \leq i<j \leq k+1-l$. By Lemma 6.10 , since $J\left(M_{k+1}\right)$ is an extension-closed subcategory of $\Lambda_{k+1}^{k+1}$, we can argue in a similar way that

$$
\operatorname{Ext}_{\bmod \Lambda_{k+1}^{k+1}}^{1}\left(X_{i}, Y_{j}\right)=\operatorname{Ext}_{\bmod \Lambda_{k+1}^{k+1}}^{1}\left(Y_{j}, X_{i}\right)=0,
$$

where $1 \leq i \leq l-1$ and $1 \leq j \leq k+1-l$. By another similar argument,

$$
\operatorname{Ext}_{\bmod \Lambda_{k+1}^{k+1}}^{1}\left(X_{j}, X_{i}\right)=0
$$

for $1 \leq i \leq j \leq l-1$ and

$$
\mathrm{Ext}_{\bmod \Lambda_{k+1}^{k+1}}^{1}\left(Y_{j}, Y_{i}\right)=0
$$

for $1 \leq i \leq j \leq k+1-l$. So we can conclude that the sequence $\left(M_{1}, M_{2}, \ldots, M_{k}\right)$ is weak exceptional in $\bmod \Lambda_{k+1}^{k+1}$, hence $M=\left(M_{1}, M_{2}, \ldots M_{k+1}\right)$ is a full weak exceptional sequence in $\bmod \Lambda_{k+1}^{k+1}$. This completes the proof.

Corollary 6.13 The complete $\tau$-exceptional sequences of $\bmod \Lambda_{n}^{n}$ and the full weak exceptional sequences of $\bmod \Lambda_{n}^{n}$ coincide.

Proof Let us denote by $T_{n}$ the set of complete $\tau$-exceptional sequences in $\bmod \Lambda_{n}^{n}$ and denote by $W_{n}$ the set of full weak exceptional sequences in $\bmod \Lambda_{n}^{n}$. Using Proposition 6.12, we can construct the following map, $f: T_{n} \rightarrow W_{n}$, where by $f(M)=M$. The map $f$ is clearly injective and since $\left|T_{n}\right|=\left|W_{n}\right|=n^{n}$, the map $f$ is bijective, but more precisely the complete $\tau$-exceptional sequences of $\bmod \Lambda_{n}^{n}$ and the full weak exceptional sequences of $\bmod \Lambda_{n}^{n}$ coincide.

Unlike $\tau$-exceptional sequences, there are no complete exceptional sequences in $\bmod \Lambda_{n}^{n}$, as we will show. In general, not much is known about exceptional sequences over the Nakayama algebras $\Lambda_{n}^{n}$.

Proposition 6.14 There are no exceptional sequences $\left(M_{1}, M_{2}, \ldots, M_{l}\right)$ in $\bmod \Lambda_{n}^{n}$ of length $l>1$. In particular, there are no complete exceptional sequences in $\bmod \Lambda_{n}^{n}$ where $n>1$.

Proof Suppose $M=\left(M_{1}, M_{2}, \ldots, M_{l}\right)$ is an exceptional sequence of length $l>1$. Every indecomposable projective module in $\bmod \Lambda_{n}^{n}$ has length $n$, so by Proposition 3.12 we have that $\operatorname{Hom}\left(P_{i}, P_{j}\right) \neq 0$ for all $1 \leq i, j \leq n$. As a consequence of this $M$ cannot contain more 
than one indecomposable projective module, in particular, if $l>1$, then $M$ must contain non-projective indecomposable modules.

Let $N$ be a non-projective indecomposable module in $\bmod \Lambda_{n}^{n}$. Observe that $N=$ $\operatorname{rad}^{k}\left(P_{i}\right)$ for some $1 \leq i \leq n$ and $1 \leq k \leq n-1$. We can further observe that the length of $N$ is $l(N)=n-k$ and that $\operatorname{top}(N)=S_{(i+k)_{n}}$. Further observe that $N$ has the following infinite sequence as its projective resolution.

$$
\text { . } \longrightarrow P_{i} \longrightarrow P_{(i+k)_{n}} \longrightarrow P_{i} \longrightarrow P_{(i+k)_{n}} \longrightarrow P_{i} \longrightarrow P_{(i+k)_{n}} \longrightarrow N \longrightarrow 0
$$

Since $N$ has length $l(N)=n-k$ and $\operatorname{top}(N)=S_{(i+k)_{n}}$, we can write $N=$ $P_{(i+k)_{n}} / \operatorname{rad}^{n-k}\left(P_{(i+k)_{n}}\right)$. By Proposition 3.12, we can observe that $\operatorname{Hom}\left(P_{i}, N\right)=0$ and $\operatorname{Hom}\left(P_{(i+k)_{n}}, N\right) \neq 0$. Therefore by applying the functor $\operatorname{Hom}(-, N)$ to the above projective resolution, we obtain the following sequence.

$$
0 \longrightarrow \operatorname{Hom}(N, N) \stackrel{f_{0}}{\longrightarrow} \operatorname{Hom}\left(P_{(i+k)_{n}}, N\right) \stackrel{f_{1}}{\longrightarrow} 0 \stackrel{f_{2}}{\longrightarrow} \operatorname{Hom}\left(P_{(i+k)_{n}}, N\right) \stackrel{f_{3}}{\longrightarrow} 0 \stackrel{f_{4}}{\longrightarrow} \ldots
$$

So we have that $\operatorname{Ext}^{2}(N, N)=\operatorname{ker}\left(f_{3}\right) / \operatorname{im}\left(f_{2}\right) \neq 0$. Which is to say any non-projective module in $\bmod \Lambda_{n}^{n}$ is not exceptional. From this we conclude that $M$ cannot contain nonprojective modules. A contradiction.

\section{The $\Gamma_{n}^{n-1}$ Case}

Let $n \geq 1$ be a positive integer. In this section we will study the combinatorics for the number of complete $\tau$-exceptional sequence in $\bmod \Gamma_{n}^{n-1}$. Recall that we denote by $A_{n}$ the linearly oriented quiver with $n$ vertices,

$1 \stackrel{\alpha_{1}}{\longrightarrow} 2 \stackrel{\alpha_{2}}{\longrightarrow} 3 \stackrel{\alpha_{3}}{\longrightarrow} \ldots \stackrel{\alpha_{n-2}}{\longrightarrow} n-1 \stackrel{\alpha_{n-1}}{\longrightarrow} n$. The algebra $\Gamma_{n}^{n-1}$ is defined to be the $\mathbb{F}$-algebra, $\mathbb{F} A_{n} / R_{Q}^{n-1}$. This is the path algebra of the quiver $A_{n}$ modulo the relation $\alpha_{1} \alpha_{2} \ldots \alpha_{n-1}=0$.

Observe the following. Let $M$ be an indecomposable module in $\bmod \Gamma_{n}^{n-1}$, then $M$ belongs to one of the following disjoint sets. The first set contains the indecomposable projective modules $P_{j}$ for $1 \leq j \leq n$. The second set contains non-projective modules of the form $M=\operatorname{rad}^{i}\left(P_{1}\right)$ where $1 \leq i \leq n-2$ and $P_{1}$ is the indecomposable projective at vertex 1 . The third set contains indecomposable modules which are neither projective or of the form $M=\operatorname{rad}^{i}\left(P_{1}\right)$ for $1 \leq i \leq n-2$. Any indecomposable module $M$ in $\bmod \Gamma_{n}^{n-1}$ has length $l(M)<n$, therefore by Proposition 6.1, every indecomposable module of $\bmod \Gamma_{n}^{n-1}$ is $\tau$-rigid.

Proposition 7.1 Let $P_{i}$ be an indecomposable projective module in $\bmod \Gamma_{n}^{n-1}$ for some $1 \leq i \leq n$. Then the $\tau$-perpendicular category of $P_{i}$ in $\bmod \Gamma_{n}^{n-1}$ is $J\left(P_{i}\right) \cong \bmod \mathbb{A}_{n-i} \oplus$ $\bmod \mathbb{A}_{i-1}$, where $\mathbb{A}_{j}$ is the hereditary type A hereditary algebra.

Proof Let $P_{i}$ be an indecomposable projective with length $1 \leq l\left(P_{i}\right) \leq n-1$. By definition the Bongartz completion $T_{P_{i}}=\mathcal{P}\left({ }^{\perp}\left(\tau P_{i}\right)\right)$. Since $P_{i}$ is projective, $\tau P_{i}=0$ therefore ${ }^{\perp}\left(\tau P_{i}\right)=\bmod \Gamma_{n}^{n-1}$, hence the Bongartz completion

$$
T_{P_{i}}=\bigoplus_{j=1}^{n} P_{j} .
$$

Thus the $\mathbb{F}$-algebra $E_{P_{i}}=\operatorname{End}_{\Gamma_{n}^{n-1}}\left(T_{P_{i}}\right)$ is precisely the algebra $\Gamma_{n}^{n-1}$, the path algebra of the quiver $A_{n}^{\mathrm{op}}$,

$$
1 \stackrel{\alpha_{2}}{\longleftarrow} 2 \stackrel{\alpha_{3}}{\longleftarrow} 3 \stackrel{\alpha_{4}}{\longleftarrow} \ldots \stackrel{\alpha_{i-1}}{\longleftarrow} i-1 \stackrel{\alpha_{i}}{\longleftarrow} i \stackrel{\alpha_{i+1}}{\longleftarrow} i+1 \stackrel{\alpha_{i+2}}{\longleftarrow} \ldots \stackrel{\alpha_{n-1}}{\longleftarrow} n-1 \stackrel{\alpha_{n}}{\longleftarrow} n
$$


modulo the relation $\alpha_{n} \alpha_{n-1} \ldots \alpha_{1}=0$. Let $A_{n}^{\text {op }(i)}$ be the quiver obtained from $A_{n}^{\text {op }}$ by removing the vertex $i$ and all arrows incident to $i$.

$$
1 \stackrel{\alpha_{2}}{\longleftarrow} 2 \stackrel{\alpha_{3}}{\longleftarrow} 3 \stackrel{\alpha_{4}}{\longleftarrow} \ldots \stackrel{\alpha_{i-1}}{\longleftarrow} i-1 \quad i+1 \stackrel{\alpha_{i+2}}{\longleftarrow} \ldots \stackrel{\alpha_{n-1}}{\longleftarrow} n-1 \stackrel{\alpha_{n}}{\longleftarrow} n
$$

The quiver $A_{n}^{\mathrm{op}(i)}$ has no relations. By Lemma 3.4, $D_{M}=E_{M} /\left\langle e_{M}\right\rangle$ is the path algebra of the quiver $A_{n}^{\mathrm{op}(i)}$. Since $D_{M}=\mathbb{F} A_{n}^{\mathrm{op}(i)}$, it follows that $J(M) \cong \bmod \mathbb{A}_{n-i} \oplus \bmod \mathbb{A}_{i-1}$ by Theorem 3.5.

Proposition 7.2 Let $M$ be an indecomposable module in $\bmod \Gamma_{n}^{n-1}$ of the form $M=$ $\operatorname{rad}^{i}\left(P_{1}\right)$ for some $1 \leq i \leq n-2$ with length $1 \leq l(M) \leq n-2$. Then the $\tau$-perpendicular category of $M$ in $\bmod \Gamma_{n}^{n-1}$ is

$$
J(M) \cong\left\{\begin{array}{ll}
\bmod \mathbb{A}_{l(M)-1} \oplus \bmod \mathbb{A}_{1} \oplus \bmod \mathbb{A}_{1} & i=1 \\
\bmod \mathbb{A}_{n-l(M)} \oplus \bmod \mathbb{A}_{l(M)-1} & i \neq 1
\end{array},\right.
$$

where $\mathbb{A}_{j}$ is the hereditary type A hereditary algebra.

Proof Consider $P_{j}$ the indecomposable projective module at the vertex $j$ in $\bmod \Gamma_{n}^{n-1}$ with $j \neq 1$. Then it is easy to see that $P_{j}=\operatorname{rad}^{j-2}\left(P_{2}\right)$ and that $P_{j}$ has length $l\left(P_{j}\right)=n-j+1$. From this it follows that $\operatorname{rad}^{q}\left(P_{j}\right)=P_{j+q}$ where $0 \leq q \leq n-j$.

Let $M=\operatorname{rad}^{i}\left(P_{1}\right)$ for some $1 \leq i \leq n-1$. We observe that $l(M)=n-i-1$ and $\operatorname{top}(M)=S_{i+1}$. In accordance to Proposition 3.11, $M$ may in fact be written as $M=P_{i+1} / \mathrm{rad}^{n-i-1}\left(P_{i+1}\right)$. Using Proposition 3.11 again, we can see that AuslanderReiten translate of $M$ is given by $\tau M=\operatorname{rad}\left(P_{i+1}\right) / \operatorname{rad}^{n-i}\left(P_{i+1}\right)=P_{i+2}$ because $\operatorname{rad}\left(P_{i+1}\right)=P_{i+2}$ and $l\left(P_{i+1}\right)=n-(i+1)+1=n-i$, hence $\operatorname{rad}^{n-i}\left(P_{i+1}\right)=0$. So we see that the only indecomposable $\Gamma_{n}^{n-1}$-modules not in ${ }^{\perp}(\tau M)$ are the projectives $P_{j}=\operatorname{rad}^{s}\left(P_{i+2}\right)$ for $0 \leq s<n-i-1$, in other words $i+2 \leq j \leq n$ since $l\left(P_{i+2}\right)=n-i-1$.

We are now in the position to determine the Ext-projectives of ${ }^{\perp}(\tau M)$ where $M=$ $\operatorname{rad}^{i}\left(P_{1}\right)$. By the above calculation, we can say that for $1 \leq j \leq i+1$, the projective $P_{j}$ is in ${ }^{\perp}(\tau M)$ hence $\operatorname{Ext}_{\Gamma_{n}^{n-1}}\left(P_{j},{ }^{\perp}(\tau M)\right)=0$.

Let $N=\operatorname{rad}^{j}\left(P_{1}\right)$ for some $j>i$. Arguing as above we can see that the only indecomposable $\Gamma_{n}^{n-1}$-modules not in ${ }^{\perp}(\tau N)$ are the indecomposable projectives $P_{m}$ where $j+2 \leq m \leq n$, so it follows that $\{X: \operatorname{Hom}(X, \tau N) \neq 0\} \subset\{X: \operatorname{Hom}(X, \tau M) \neq 0\}$. This implies that $\operatorname{Ext}_{\Gamma_{n}^{n-1}}(N, X) \cong \operatorname{DHom}_{\Gamma_{n}^{n-1}}(X, \tau N)=0$ for all $X$ in ${ }^{\perp}(\tau M)$ by the Auslander-Reiten formula. Hence $N=\operatorname{rad}^{j}\left(P_{1}\right)$ is an Ext-projective in $\perp^{\perp}(\tau M)$.

For every other indecomposable $\Gamma_{n}^{n-1}$ module $Y$, we have that $\tau Y$ is in ${ }^{\perp}(\tau M)$, therefore since $\operatorname{Ext}_{\Gamma_{n}^{n-1}}(Y, \tau Y) \cong \overline{\mathrm{DHom}}_{\Gamma_{n}^{n-1}}(X, \tau N) \neq 0$. Therefore these modules are not Extprojective in ${ }^{\perp}(\tau M)$. By definition $T_{M}=\mathcal{P}\left({ }^{\perp}(\tau M)\right)$, so by the above arguments,

$$
\mathcal{P}\left({ }^{\perp}(\tau M)\right)=M \oplus \bigoplus_{s=i+1}^{n-2} \operatorname{rad}^{s}\left(P_{1}\right) \oplus \bigoplus_{j=1}^{i+1} P_{j}
$$

In the case when $i \neq 1$ the $\mathbb{F}$-algebra $E_{M}=\operatorname{End}_{\Gamma_{n}^{n-1}}\left(T_{M}\right)$ is the path algebra of the quiver $Q_{n}$,

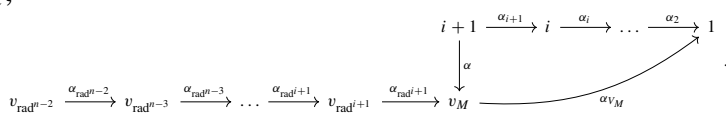


By Lemma 3.4, $D_{M}=E_{M} /\left\langle e_{M}\right\rangle$ is the path algebra of the quiver $Q_{n}^{\left(v_{M}\right)}$ which is the quiver obtained from $Q_{n}$ by removing the vertex $v_{M}$ and all arrows incident to $v_{M}$. The quiver $Q_{n}^{\left(v_{M}\right)}$ has two connected components.

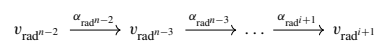

Since $D_{M}=\mathbb{F} Q_{n}^{\left(v_{M}\right)}$, it follows that $J(M) \cong \bmod \mathbb{A}_{i+1} \oplus \bmod \mathbb{A}_{n-i-2}$ by Theorem 3.5. Recall that $l(M)=n-i-1$, hence $J(M) \cong \bmod \mathbb{A}_{n-l(M)} \oplus \bmod \mathbb{A}_{l(M)-1}$.

When $i=1$ however, the $\mathbb{F}$-algebra $E_{M}=\operatorname{End}_{\Gamma_{n}^{n-1}}\left(T_{M}\right)$ is the path algebra of the quiver $Q_{n}^{\prime}$,

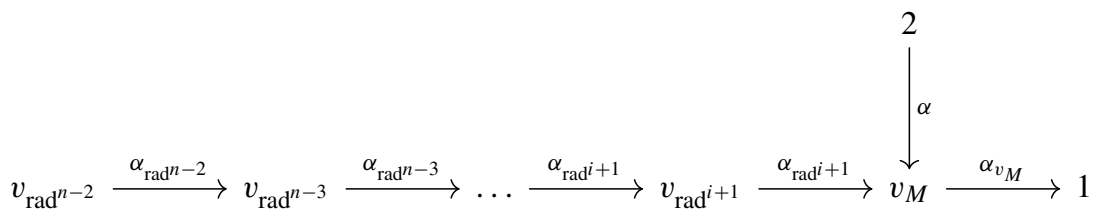

with no relations. By Lemma, 3.4 $D_{M}=E_{M} /\left\langle e_{M}\right\rangle$ is the path algebra of the quiver $Q_{n}^{\prime\left(v_{M}\right)}$ which is the quiver obtained from $Q_{n}^{\prime}$ by removing the vertex $v_{M}$ and all arrows incident to $v_{M}$. This quiver has three connected components.

$$
v_{\mathrm{rad}^{n-2}} \stackrel{\alpha_{\mathrm{rad}^{n-2}}}{\longrightarrow} v_{\mathrm{rad}^{n-3}} \stackrel{\alpha_{\mathrm{rad}^{n-3}}}{\longrightarrow} \ldots \stackrel{\alpha_{\mathrm{rad}^{i+1}}}{\longrightarrow} v_{\mathrm{rad}^{i+1}}
$$

Since $D_{M}=\mathbb{F} Q_{n}^{\prime\left(v_{M}\right)}$, it follows that $J(M) \cong \bmod \mathbb{A}_{n-3} \oplus \bmod \mathbb{A}_{1} \oplus \bmod \mathbb{A}_{1}$ by Theorem 3.5. Since $l\left(\operatorname{rad}^{1}\left(P_{1}\right)\right)=n-2$ then $J(M) \cong \bmod \mathbb{A}_{l(M)-1} \oplus \bmod \mathbb{A}_{1} \oplus \bmod \mathbb{A}_{1}$.

Proposition 7.3 Let $M$ be an indecomposable $\Gamma_{n}^{n-1}$-module such that $M \neq \operatorname{rad}^{k}(P)$ for some indecomposable projective $P$ and positive integer $k$. Suppose $M$ has length $1 \leq l(M) \leq n-2$, then the $\tau$-perpendicular category of $M$ in $\bmod \Gamma_{n}^{n-1}$ is $J(M) \cong$ $\bmod \mathbb{A}_{l(M)-1} \oplus \bmod \Gamma_{n-l(M)}^{n-l(M)-1}$.

Proof By Proposition 3.11, we can write $M=P_{i} / \mathrm{rad}^{l(M)}\left(P_{i}\right)$ for some $1 \leq i \leq n-1$ and $\tau M=\operatorname{rad}\left(P_{i}\right) / \operatorname{rad}^{l(M)+1}\left(P_{i}\right)$. We will consider the case where $i \neq 1$ and $i=1$ separately.

Suppose $i \neq 1$, then we have that $P_{i}=\operatorname{rad}^{i-2}\left(P_{2}\right)$ and $l\left(P_{i}\right)=n-i+1$. Therefore, $\operatorname{rad}^{q}\left(P_{i}\right)=P_{i+q}$, in particular we have that $\operatorname{rad}\left(P_{i}\right)=P_{i+1}$, hence $\tau M=$ $P_{i+1} / \mathrm{rad}^{l(M)}\left(P_{i+1}\right)$. Now suppose that $i=1$, hence $M=P_{1} / \operatorname{rad}^{l(M)}\left(P_{1}\right)$, then $\tau M=$ $\operatorname{rad}\left(P_{1}\right) / \operatorname{rad}^{l(M)+1}\left(P_{1}\right)$. Observe that $\operatorname{top}(\tau M)=S_{2}$ and $l(\tau M)=l(M)$, hence $\tau M=$ $P_{2} / \operatorname{rad}^{l(M)}\left(P_{2}\right)$. In either case of $i$, we have that $\tau M=P_{i+1} / \mathrm{rad}^{l(M)}\left(P_{i+1}\right)$.

Now let $X=P_{j} / \operatorname{rad}^{l}\left(P_{j}\right)$ be an arbitrary indecomposable $\Gamma_{n}^{n-1}$ module. By Proposition $3.12, \operatorname{Hom}(X, \tau M) \neq 0$ if and only $j \in[i+1, i+l(M)]_{n}$ and $i+l(M) \in[j, j+l-1]_{n}$. From this it follows that $P_{j}$ is not in ${ }^{\perp}(\tau M)$ if $i+1 \leq j \leq i+l(M)$. Hence $\operatorname{Ext}_{\Gamma_{n}^{n-1}}\left(P_{j},{ }^{\perp}(\tau M)\right)=$ 0 if $j \notin[i+1, i+l(M)]$.

Consider the module $\operatorname{rad}^{s}(M)$ for $1 \leq s \leq l(M)-1$. The length of $\operatorname{rad}^{s}(M)$ is given by $l\left(\operatorname{rad}^{s}(M)\right)=l(M)-s$. Moreover, $\operatorname{rad}^{s}(M)=P_{i+s} / \operatorname{rad}^{l(M)-s}\left(P_{i+s}\right)$, from which it follows that $\tau \operatorname{rad}^{s}(M)=P_{i+s+1} / \operatorname{rad}^{l(M)-s}\left(P_{i+s+1}\right)$. Again let $X=P_{j} / \operatorname{rad}^{l}\left(P_{j}\right)$ be an 
arbitrary indecomposable $\Gamma_{n}^{n-1}$ module. By Proposition 3.12, $\operatorname{Hom}\left(X, \tau \operatorname{rad}^{S}(M)\right) \neq 0$ if and only $j \in[i+s+1, i+l(M)]_{n}$ and $i+l(M) \in[j, j+l-1]_{n}$. Therefore $\left\{X: \operatorname{Hom}\left(X, \tau \operatorname{rad}^{s}(M)\right) \neq 0\right\} \subset\{X: \operatorname{Hom}(X, \tau M) \neq 0\}$, which implies that $\operatorname{Ext}_{\Gamma_{n}^{n-1}}\left(\operatorname{rad}^{s}(M), Y\right) \cong \overline{\mathrm{Hom}}_{\Gamma_{n}^{n-1}}\left(Y, \tau \operatorname{rad}^{S}(M)\right)=0$ for all $Y$ in ${ }^{\perp}(\tau M)$. In other words, $\operatorname{rad}^{S}(M)$ is Ext-projective in ${ }^{\perp}(\tau M)$.

By Proposition 3.1, $M$ is Ext-projective in ${ }^{\perp}(\tau M)$, so

$$
\mathcal{P}\left({ }^{\perp}(\tau M)\right)=M \oplus \bigoplus_{s=1}^{l(M)-1} \operatorname{rad}^{s}(M) \oplus \bigoplus_{j \notin[i+1, i+l(M)]} P_{j} .
$$

By definition, the Bongartz completion $T_{M}=\mathcal{P}\left({ }^{\perp}(\tau M)\right)$, so the $\mathbb{F}$-algebra $E_{M}=$ End $_{\Gamma_{n}^{n-1}}\left(T_{M}\right)$ is the path algebra of the quiver $Q_{n}$ modulo relations (set $l(M):=m$ ),

$$
\begin{gathered}
n \stackrel{\alpha_{n}}{\longrightarrow} n-1 \stackrel{\alpha_{n-1}}{\longrightarrow} \ldots \stackrel{\alpha_{i+m+2}}{\longrightarrow} i+m+1 \stackrel{\alpha_{i+m+1}}{\longrightarrow} i \stackrel{\alpha_{i}}{\longrightarrow} i-1 \stackrel{\alpha_{i-1}}{\longrightarrow} \ldots \stackrel{\alpha_{2}}{\longrightarrow} 1 \\
v_{\mathrm{rad}^{m-1}} \stackrel{\alpha_{\mathrm{rad}^{m-1}}}{\longrightarrow} v_{\mathrm{rad}^{m-2}} \stackrel{\alpha_{\mathrm{rad}^{m-2}}}{\longrightarrow} \ldots \stackrel{\alpha_{\mathrm{rad}^{2}}}{\longrightarrow} v_{\mathrm{rad}^{1}} \stackrel{\alpha_{\mathrm{rad}^{1}}}{\longrightarrow} v_{M}^{\mid}
\end{gathered}
$$

Since the vertices of the top row of the quiver correspond to the indecomposable projectives of $\bmod \Gamma_{n}^{n-1}$ and the arrows reflect the relations the corresponding maps between the projectives, we see that we have the relation $\alpha_{n} \alpha_{n-1} \ldots \alpha_{i+m+1} \alpha_{i} \alpha_{i-1} \ldots \alpha_{1}=0$. Let $Q_{n}^{\left(v_{M}\right)}$ be the quiver obtained from $Q_{n}$ by removing the vertex $v_{M}$ and all the arrows incident to $v_{M}$,

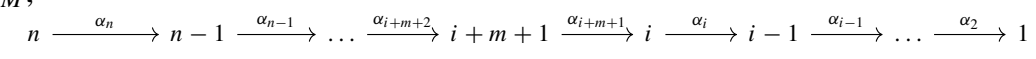

$$
v_{\mathrm{rad}^{m-1}} \stackrel{\alpha_{\mathrm{rad}^{m-1}}}{\longrightarrow} v_{\mathrm{rad}^{m-2}} \stackrel{\alpha_{\mathrm{rad}}{ }^{m-2}}{\longrightarrow} \ldots \stackrel{\alpha_{\mathrm{rad}^{2}}}{\longrightarrow} v_{\mathrm{rad}^{1}}
$$

with the relation $\alpha_{n} \alpha_{n-1} \ldots \alpha_{i+m+1} \alpha_{i} \alpha_{i-1} \ldots \alpha_{1}=0$. By Lemma 3.4, $D_{M}=E_{M} /\left\langle e_{M}\right\rangle$ is the path algebra of the quiver $Q_{n}^{\left(v_{M}\right)}$ modulo the relation $\alpha_{n} \alpha_{n-1} \ldots \alpha_{i+m+1} \alpha_{i} \alpha_{i-1} \ldots \alpha_{1}=$ 0 . It follows that $J(M) \cong \bmod \mathbb{A}_{l(M)-1} \oplus \bmod \Gamma_{n-l(M)}^{n-l(M)-1}$ by Theorem 3.5.

Theorem 7.4 Let $K_{n}$ denote the number of complete $\tau$-exceptional sequences in $\bmod \Gamma_{n}^{n-1}$. Then $K_{n}$ satisfies the recurrence relation;

$$
\begin{gathered}
K_{n}=(n-1)(n-2)^{(n-3)}+\sum_{i=1}^{n}\left(\begin{array}{c}
n-1 \\
i-1
\end{array}\right)(n-i+1)^{(n-i-1)} i^{i-2}+\sum_{i=1}^{n-3}\left(\begin{array}{c}
n-1 \\
i-1
\end{array}\right)(n-i+1)^{(n-i-1)} i^{i-2} \\
+\sum_{i=1}^{n-2}\left(\begin{array}{c}
n-1 \\
i-1
\end{array}\right)(n-i-1) i^{i-2} K_{n-i}
\end{gathered}
$$

with $K_{1}=1$.

Proof Let $M$ be an indecomposable module in $\bmod \Gamma_{n}^{n-1}$. Suppose $\left(X_{1}, X_{2}, \ldots, X_{n-1}, M\right)$ is a $\tau$-exceptional sequence in $\bmod \Gamma_{n}^{n-1}$. Then by definition and the fact that $\delta(J(M))=$ $n-1$, the sequence $\left(X_{1}, X_{2}, \ldots, X_{n-1}\right)$ is a complete $\tau$-exceptional sequence in $J(M)$. Hence the number of complete $\tau$-exceptional sequences ending in $M$ is equal to the number of complete $\tau$-exceptional sequences in $J(M)$.

Suppose $M$ is projective, hence $M=P_{i}$ for some $1 \leq i \leq n$, then by Proposition 7.1 the $\tau$-perpendicular category $J(M) \cong \bmod \mathbb{A}_{n-i} \oplus \bmod \mathbb{A}_{i-1}$. The number of complete $\tau$ exceptional sequences in mod $\mathbb{A}_{l}$ is precisely the number of complete exceptional sequence 
in $\bmod \mathbb{A}_{l}$ which is shown in [[28] [Proposition 1.1]] to be $(l+1)^{(l-1)}$. Therefore by Theorems 3.8 and 3.9 the number of complete $\tau$-exceptional sequence ending in $M=P_{i}$ is $\left(\begin{array}{c}n-1 \\ i-1\end{array}\right)(n-i+1)^{n-i-1} i^{i-2}$.

Suppose $M=\operatorname{rad}^{i}\left(P_{1}\right)$ for some $1 \leq i \leq n-2$. If $i=1$ then we saw in Proposition 7.2 that $J(M) \cong \bmod \mathbb{A}_{n-3} \oplus \bmod \mathbb{A}_{1} \oplus \bmod \mathbb{A}_{1}$. Arguing as above it follows that the number of complete $\tau$-exceptional sequences ending in $\operatorname{rad}^{1}\left(P_{1}\right)$ is $\left(\begin{array}{c}n-1 \\ n-3,1,1\end{array}\right)(n-2)^{(n-4)} 2^{0} 2^{0}=(n-$ $1)(n-2)^{(n-3)}$. If it is the case that $2 \leq i \leq n-2$, then $J(M) \cong \bmod \mathbb{A}_{n-l(M)} \oplus \bmod \mathbb{A}_{l(M)-1}$. Therefore the number of complete $\tau$-exceptional sequences ending in $M=\operatorname{rad}^{i}\left(P_{1}\right)$ for some $2 \leq i \leq n-2$ is $\left(\begin{array}{c}n-1 \\ l(M)-1\end{array}\right)(n-l(M)+1)^{n-l(M)-1} l(M)^{l(M)-2}$, where $l(M)$ is the length of $M$.

Finally suppose that $M$ is not of the form $\operatorname{rad}^{i}(P)$ for some indecomposable projective module $P$. By Proposition 7.3, $J(M) \cong \bmod \mathbb{A}_{l(M)-1} \oplus \bmod \Gamma_{n-l(M)}^{n-l(M)-1}$ where $l(M)$ is the length of $M$. Therefore the number of complete $\tau$-exceptional sequences ending in $M$ is $\left(\begin{array}{c}n-1 \\ l(M)-1\end{array}\right) K_{n-l(M)} l(M)^{l(M)-2}$. Observe that in this case the length of $M$ is $1 \leq l(M) \leq n-2$ and for each fixed value of $l(M)$ there are $n-l(M)-1$ indecomposable modules $M$ such that $M \neq \operatorname{rad}^{i}(P)$.

By counting the number of complete $\tau$-exceptional sequences ending in each indecomposable $\Gamma_{n}^{n-1}$-module $M$, the recurrence relation of $K_{n}$ follows. It is also trivial to see that $K_{1}=1$.

Theorem 7.5 Let $h(x)=\sum_{n=0}^{\infty} K_{n} \frac{x^{n}}{n !}$ be the exponential generating function of $K_{n}$. Then $h(x)$ satisfies the first order linear $O D E$,

$$
h^{\prime}(x)\left(1-x e^{-W(-x)}\right)+h(x) e^{-W(-x)}=2 e^{-2 W(-x)}-e^{-W(-x)}+W(-x)+\frac{1}{2} x W(-x) .
$$

Proof Let $h(x)=\sum_{n=0}^{\infty} K_{n} \frac{x^{n}}{n !}$ be the exponential generating function of $K_{n}$. Let $a(n)=$ $(n+1)^{n-1}$. Let $g(x)=\sum_{n=0}^{\infty}(n+1)^{n-1} \frac{x^{n}}{n !}$. Then $g(x)=e^{-W(-x)}$ by Lemma 6.8 , where $W(x)$ is Lambert's $W$ function. By the only Proposition in Section 6 of [22],

$$
2(n+2)^{n-1}=\sum_{i=0}^{n}\left(\begin{array}{l}
n \\
i
\end{array}\right) a(i) a(n-i) .
$$

So it follows from Lemma 6.7 that

$$
(g(x))^{2}=\sum_{n=0}^{\infty} 2(n+2)^{n-1} \frac{x^{n}}{n !} .
$$

We make the following observations about

$$
\sum_{i=1}^{n}\left(\begin{array}{c}
n-1 \\
i-1
\end{array}\right)(n-i+1)^{(n-i-1)} \cdot i^{i-2} .
$$

With the change of variable $j=i-1$,

$$
\sum_{i=1}^{n}\left(\begin{array}{c}
n-1 \\
i-1
\end{array}\right)(n-i+1)^{(n-i-1)} \cdot i^{i-2}=\sum_{j=0}^{n-1}\left(\begin{array}{c}
n-1 \\
j
\end{array}\right)(n-j)^{(n-j-2)}(j+1)^{j-1}=2(n+1)^{n-2},
$$


as shown in the proof of the only Proposition in Section 6 of [22]. We also observe that

$\sum_{i=1}^{n}\left(\begin{array}{c}n-1 \\ i-1\end{array}\right)(n-i+1)^{(n-i-1)} \cdot i^{i-2}=\sum_{i=1}^{n-3}\left(\begin{array}{c}n-1 \\ i-1\end{array}\right)(n-i+1)^{(n-i-1)} \cdot i^{i-2}+n^{n-2}+(n-1)^{n-2}+\frac{3}{2}(n-1)(n-2)^{n-3}$,

so,

$\sum_{i=1}^{n-3}\left(\begin{array}{c}n-1 \\ i-1\end{array}\right)(n-i+1)^{(n-i-1)} \cdot i^{i-2}=2(n+1)^{n-2}-n^{n-2}-(n-1)^{n-2}-\frac{3}{2}(n-1)(n-2)^{n-3}$.

As a result we can write the recurrence for $K_{n+1}$ (from Theorem 7.4) in the following way,

$K_{n+1}=2 \cdot 2(n+2)^{n-1}-(n+1)^{n-1}-n^{n-1}-\frac{1}{2}(n)(n-1)^{n-2}+\sum_{i=1}^{n-1}\left(\begin{array}{c}n \\ i-1\end{array}\right)(n-i) K_{n+1-i} \cdot i^{i-2}$.

Making the change of variable $j=i-1$ we get,

$K_{n+1}=2 \cdot 2(n+2)^{n-1}-(n+1)^{n-1}-n^{n-1}-\frac{1}{2}(n)(n-1)^{n-2}+\sum_{j=0}^{n-2}\left(\begin{array}{l}n \\ j\end{array}\right)(n-j-1) K_{n-j} \cdot(j+1)^{j-1}$.

We will now study the exponential generating function of $K_{n+1}$. To do this we look at the exponential generating function of each of the summands on the right hand side. We have already seen from Eq. 1 that

$$
\sum_{n=0}^{\infty} 2(n+2)^{n-1} \frac{x^{n}}{n !}=(g(x))^{2}
$$

To deal with the rest of the summands of $K_{n+1}$ in Eq. 3 but the last one, we first re-organise them in the following way using Eq. 2. Let

$\phi(n)=\sum_{i=1}^{n-2}\left(\begin{array}{c}n-1 \\ i-1\end{array}\right)(n-i+1)^{(n-i-1)} \cdot i^{i-2}=2(n+2)^{n-1}-(n+1)^{n-1}-n^{n-1}-\frac{3}{2} n(n-1)^{n-2}$.

The change of variable $j=i-1$ gives us

$$
\phi(n)=\sum_{j=0}^{n-3}\left(\begin{array}{l}
n \\
j
\end{array}\right)(n-j)^{n-j-2}(j+1)^{j-1} .
$$

We have $\phi(n)=0$ for $n=0,1,2$ since the sum is empty for these values of $n$. This further implies that,

$$
\begin{gathered}
\sum_{n=0}^{\infty} \phi(n) \frac{x^{n}}{n !}=\sum_{n=2}^{\infty} \phi(n) \frac{x^{n}}{n !} \\
=\sum_{n=2}^{\infty} 2(n+2)^{n-1} \frac{x^{n}}{n !}-\sum_{n=2}^{\infty}(n+1)^{n-1} \frac{x^{n}}{n !}-\sum_{n=2}^{\infty} n^{n-1} \frac{x^{n}}{n !}-\frac{3}{2} \sum_{n=2}^{\infty} n(n-1)^{n-2} \frac{x^{n}}{n !} \\
=\left((g(x))^{2}-1-2 x\right)-\sum_{n=2}^{\infty}(n+1)^{n-1} \frac{x^{n}}{n !}-\sum_{n=2}^{\infty} n^{n-1} \frac{x^{n}}{n !}-\frac{3}{2} \sum_{n=2}^{\infty} n(n-1)^{n-2} \frac{x^{n}}{n !},
\end{gathered}
$$


by Eq. 1. Lemma 6.8 resolves the second summand. The third summand is resolved by [9] in Section 2, page 4. This was previously was done in [24]. This has been translated into English; see [23, Page 48]). To resolve the fourth summand we use the fact the exponential generating function is a right index shift and multiplication by $n$ of the $3 \mathrm{rd}$ summand. Right index shifting is equivalent to formal integration and by Rule $2^{\prime}$ in Section 2.3 page 41 of [31] multiplication by $n$ is equivalent to differentiating and then multiplying the exponential generating function by $x$ (This is also given on the OEIS A055541). Therefore.

$$
\begin{gathered}
\sum_{n=0}^{\infty} \phi(n) \frac{x^{n}}{n !}=\left[e^{-2 W(-x)}-1-2 x\right]-\left[e^{-W(-x)}-1-x\right]-[-W(-x)-x]-\frac{3}{2}[-x W(-x)] \\
=e^{-2 W(-x)}-1-2 x-e^{-W(-x)}+1+x+W(-x)+x+\frac{3}{2} x W(-x) \\
=e^{-2 W(-x)}-e^{-W(-x)}+W(-x)+\frac{3}{2} x W(-x)
\end{gathered}
$$

Now let us study the final summand of Eq. 3

$$
\sum_{j=0}^{n-2}\left(\begin{array}{l}
n \\
j
\end{array}\right)(n-j-1) K_{n-j} \cdot(j+1)^{j-1} .
$$

Notice that the term $\left(\begin{array}{c}n \\ n-1\end{array}\right)(n-(n-1)-1) K_{1} n^{n-2}=0$ and $\left(\begin{array}{l}n \\ n\end{array}\right)(n-n-1) K_{0} n+1^{n-1}=0$ since $K_{0}=0$. Therefore,

$$
\sum_{j=0}^{n-2}\left(\begin{array}{l}
n \\
j
\end{array}\right)(n-j-1) K_{n-j} \cdot(j+1)^{j-1}=\sum_{j=0}^{n}\left(\begin{array}{c}
n \\
j
\end{array}\right)(n-j-1) K_{n-j} \cdot(j+1)^{j-1} .
$$

By Lemma 6.7,

$$
\sum_{n=0}^{\infty}\left(\sum_{j=0}^{n}\left(\begin{array}{l}
n \\
j
\end{array}\right)(n-j-1) K_{n-j} \cdot(j+1)^{j-1}\right) \frac{x^{n}}{n !}=\left(\sum_{n=0}^{\infty}(n-1) K_{n} \frac{x^{n}}{n !}\right)\left(\sum_{n=0}^{\infty}(n+1)^{n-1} \frac{x^{n}}{n !}\right) .
$$

By Rule $2^{\prime}$ in Section 2.3 page 41 of [31]

$$
\left(\sum_{n=0}^{\infty}(n-1) K_{n} \frac{x^{n}}{n !}\right)=x \frac{d}{d x}\left(\sum_{n=0}^{\infty} K_{n} \frac{x^{n}}{n !}\right)-\left(\sum_{n=0}^{\infty} K_{n} \frac{x^{n}}{n !}\right)=x h^{\prime}(x)-h(x) .
$$

By Lemma 6.8,

$$
\left(\sum_{n=0}^{\infty}(n+1)^{n-1} \frac{x^{n}}{n !}\right)=e^{-W(-x)}
$$

Therefore

$$
\sum_{n=0}^{\infty}\left(\sum_{j=0}^{n}\left(\begin{array}{l}
n \\
j
\end{array}\right)(n-j-1) K_{n-j} \cdot(j+1)^{j-1}\right) \frac{x^{n}}{n !}=\left(x h^{\prime}(x)-h(x)\right) e^{-W(-x)} .
$$

By Rule 1' in Section 2.3 page 41 of [31],

$$
\sum_{n=0}^{\infty} K_{n+1} \frac{x^{n}}{n !}=h^{\prime}(x)
$$


We now write the exponential generating function of $K_{n+1}$, using the expression of $K_{n+1}$ in Eq. 3 and the exponential generating functions of the summands of $K_{n+1}$ obtained as in Eqs. 4, 5 and 6.

$\sum_{n=0}^{\infty} K_{n+1} \frac{x^{n}}{n !}=2 e^{-2 W(-x)}-e^{-W(-x)}+W(-x)+\frac{1}{2} x W(-x)+\left(x h^{\prime}(x)-h(x)\right) e^{-W(-x)}$,

so

$$
h^{\prime}(x)=2 e^{-2 W(-x)}-e^{-W(-x)}+W(-x)+\frac{1}{2} x W(-x)+\left(x h^{\prime}(x)-h(x)\right) e^{-W(-x)},
$$

Therefore we have the following first order linear ODE,

$$
h^{\prime}(x)+\frac{h(x) e^{-W(-x)}}{\left(1-x e^{-W(-x)}\right)}=\frac{2 e^{-2 W(-x)}-e^{-W(-x)}+W(-x)+\frac{1}{2} x W(-x)}{\left(1-x e^{-W(-x)}\right)} .
$$

This ODE is of the form,

$$
h^{\prime}(x)+Q(x) h(x)=F(x),
$$

so we may apply the integrating factor method and give a general solution for $h(x)$,

$$
h(x)=e^{-V(x)} \int V(x) F(x) d x+C,
$$

where $V(X)$ is the integrating factor,

$$
V(X)=\int Q(x) d x=\int \frac{e^{-W(-x)}}{1-x e^{-W(-x)}} d x .
$$

Unfortunately, we are unable to evaluate $V(X)$ so we leave $h(x)$ as it is.

\section{Justification}

In this section we would like to justify why we only look at the four cases above. Our approach to counting the number of complete $\tau$-exceptional sequences in the above module categories relied upon Theorems 3.8 and 3.9. We also took advantage of the fact that the $\tau$ perpendicular categories of indecomposable modules $M$ were of the form $J(M) \cong \mathcal{C} \oplus \mathcal{D}$ with $\mathcal{C}$ and $\mathcal{D}$ being module categories in the the two families $\Gamma_{n}^{t}$ or $\Lambda_{n}^{t}$. It is our claim that these four cases, $\Gamma_{n}^{2}, \Gamma_{n}^{n-1}, \Lambda_{n}^{2}, \Lambda_{n}^{n}$ are the only ones were all the $\tau$-perpendicular categories $J(M)$ are of this form. In other words, our approach only works on these four cases.

Proposition 8.1 Fix a positive integers $t \geq 3$. For $n \geq t+1$, let $A=\Lambda_{n}^{t}$. Then there exists an A-module $M$ such that the $\tau$-perpendicular category $J(M)$ is not a direct sum of module categories over algebras of the form $\Lambda_{n^{\prime}}^{t^{\prime}}$ or $\Gamma_{n^{\prime}}^{t^{\prime}}$ for $2 \leq t^{\prime} \leq n^{\prime}<n$.

Proof We prove this by counter-example. Set $M=S_{1}$, the simple module at vertex 1 of the quiver $C_{n}$ of $A$. Note that other simple modules also work, but for simplicity we choose $S_{1}$. The Auslander-Reiten translate of $S_{1}$ is $\tau S_{1}=S_{2}$. Using Proposition 3.11 and 3.12, we can say that $\operatorname{Hom}\left(X, S_{2}\right) \neq 0$ if and only if $X=P_{2} / \operatorname{rad}^{l(X)}\left(P_{2}\right)$ where $l(X)$ is the length of $X$. It also follows that $P_{2}$ is the only projective with non-zero maps to $S_{2}$. Therefore all other indecomposable projective modules $P_{j}$ with $j \neq 2$ are in ${ }^{\perp}\left(\tau S_{1}\right)$, hence they are Extprojectives in ${ }^{\perp}\left(\tau S_{1}\right)$. By Proposition 3.1, the module $S_{1}$ is Ext-project in ${ }^{\perp}\left(\tau S_{1}\right)$. We can 
thus conclude that,

$$
\mathcal{P}\left({ }^{\perp}\left(\tau S_{1}\right)\right)=\bigoplus_{j \neq 2} P_{j} \oplus S_{1} .
$$

By definition the Bongartz completion of $M$ in $\bmod A$ is $T_{M}=\mathcal{P}\left(\tau S_{1}\right)$. Let $Q_{n}$ be the following quiver,

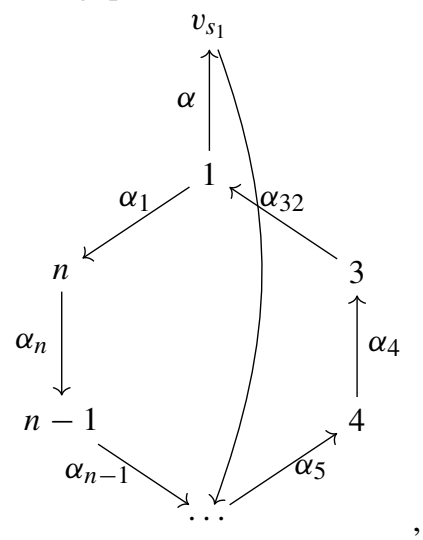

where the vertices labelled $j$ correspond to the projective $P_{j}$ and the vertex $v_{s_{1}}$ corresponds to the simple $S_{1}$ and the arrows correspond to the irreducible maps between their respective modules. The $\mathbb{F}$-algebra $E_{M}=\operatorname{End}_{A}\left(T_{M}\right)$ is the path algebra of the quiver modulo relations. Let $Q_{n}^{v_{s_{1}}}$ be the quiver obtained from $Q_{n}$ by removing the vertex $v_{s_{1}}$ and any arrows incident to $v_{s_{1}}$,

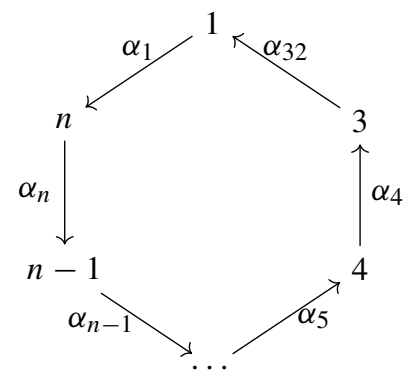

by Lemma 3.4, $D_{M}=E_{M} /\left\langle e_{M}\right\rangle$ is the path algebra of the quiver $Q_{n}^{v_{s_{1}}}$ modulo relations. We have the relation $\alpha_{t+1} \alpha_{t} \ldots \alpha_{4} \alpha_{32}=0$ involving $t-1$ arrows because it corresponds to $\operatorname{Hom}_{A}\left(P_{t+1}, P_{2}\right)=0$ since in $\bmod \Lambda_{n}^{t}$ the composition of $t$ maps between projectives is 0 . However, at the same time we have that the composition of the $t^{\prime}-1$ arrows $\alpha_{1} \alpha_{n} \ldots \alpha_{n-\left(t^{\prime}-3\right)} \neq 0$ for $2 \leq t^{\prime} \leq t$. Therefore as a module category $J(M)$ cannot be a direct sum of module categories of the form $\bmod \Lambda_{n^{\prime}}^{t^{\prime}}$ or $\bmod \Gamma_{n^{\prime}}^{t^{\prime}}$ as required.

Proposition 8.2 Fix a positive integers $t \geq 3$. For $n \geq t+2$, let $A=\Gamma_{n}^{t}$. Then there exists an $A$-module $M$ such that the $\tau$-perpendicular category $J(M)$ is not a direct sum of module categories over algebras of the form $\Gamma_{n^{\prime}}^{t^{\prime}}$ or $\Lambda_{n^{\prime}}^{t^{\prime}}$ for $2 \leq t^{\prime} \leq n^{\prime}<n$.

Proof The argument is similar to that for the previous proposition. We prove this by counterexample. Set $M=S_{1}$, the simple module at vertex 1 of the quiver $A_{n}$ of $A$. The AuslanderReiten translate of $S_{1}$ is $\tau S_{1}=S_{2}$. By Proposition 3.11 and 3.12, $\operatorname{Hom}\left(X, S_{2}\right) \neq 0$ if and only if $X=P_{2} / \operatorname{rad}^{l(X)}\left(P_{2}\right)$ where $l(X)$ is the length of $X$. It also follows that $P_{2}$ is the only 
projective with non-zero maps to $S_{2}$. Therefore all other indecomposable projective modules $P_{j}$ with $j \neq 2$ are in ${ }^{\perp}\left(\tau S_{1}\right)$, hence they are Ext-projectives in ${ }^{\perp}\left(\tau S_{1}\right)$. By Proposition 3.1, the module $S_{1}$ is Ext-project in ${ }^{\perp}\left(\tau S_{1}\right)$ We can thus conclude that,

$$
\mathcal{P}\left(\tau S_{1}\right)=\bigoplus_{j \neq 2} P_{j} \oplus S_{1}
$$

By definition the Bongartz completion of $M$ in $\bmod A$ is $T_{M}=\mathcal{P}\left(\tau S_{1}\right)$. Let $Q_{n}$ be the following quiver,

$$
v_{s_{1}} \stackrel{\alpha}{\longleftarrow} 1 \stackrel{\alpha_{32}}{\longleftarrow} 3 \stackrel{\alpha_{4}}{\longleftarrow} 4 \stackrel{\alpha_{5}}{\longleftarrow} \ldots \stackrel{\alpha_{n-1}}{\longleftarrow} n-1 \stackrel{\alpha_{n}}{\longleftarrow} n,
$$

where the vertices labelled $j$ correspond to the projective $P_{j}$ and the vertex $v_{s_{1}}$ corresponds to the simple $S_{1}$ and the arrows correspond to the irreducible maps between their respective modules. The $\mathbb{F}$-algebra $E_{M}=\operatorname{End}_{A}\left(T_{M}\right)$ is the path algebra of the quiver $Q_{n}$ modulo relations. Let $Q_{n}^{v_{s_{1}}}$ be the quiver obtained from $Q_{n}$ by removing the vertex $v_{s_{1}}$ and any arrows incident to $v_{s_{1}}$,

$$
1 \stackrel{\alpha_{32}}{\longleftarrow} 3 \stackrel{\alpha_{4}}{\longleftarrow} 4 \stackrel{\alpha_{5}}{\longleftarrow} \ldots \stackrel{\alpha_{n-1}}{\longleftarrow} n-1 \stackrel{\alpha_{n}}{\longleftarrow} n .
$$

by Lemma 3.4, $D_{M}=E_{M} /\left\langle e_{M}\right\rangle$ is the path algebra of the quiver $Q_{n}^{v_{s_{1}}}$ modulo relations. We have the relation $\alpha_{t+1} \alpha_{t} \ldots \alpha_{4} \alpha_{32}=0$ involving $t-1$ arrows because it corresponds to $\operatorname{Hom}_{A}\left(P_{t+1}, P_{2}\right)=0$ since in $\bmod \Gamma_{n}^{t}$ the composition of $t$ maps between projectives is 0 . However, at the same time we have that the composition of the $t^{\prime}-1$ arrows $\alpha_{n} \alpha_{n-1} \ldots \alpha_{n-\left(t^{\prime}-2\right)} \neq 0$. Therefore as a module category $J(M)$ cannot be a direct sum of module categories of the form $\bmod \Lambda_{n^{\prime}}^{t^{\prime}}$ or $\bmod \Gamma_{n^{\prime}}^{t^{\prime}}$ as required.

So we have shown that our strategy for deriving recurrences for the number of complete $\tau$-exceptional sequences over Nakayama algebras only works in the four cases we've studied. However, the statements of Theorems 3.8 and 3.9 are general enough that a similar strategy may be applied to other algebras, and may prove as effective for counting the $\tau$-exceptional sequences for the module categories of those algebras.

Open Access This article is licensed under a Creative Commons Attribution 4.0 International License, which permits use, sharing, adaptation, distribution and reproduction in any medium or format, as long as you give appropriate credit to the original author(s) and the source, provide a link to the Creative Commons licence, and indicate if changes were made. The images or other third party material in this article are included in the article's Creative Commons licence, unless indicated otherwise in a credit line to the material. If material is not included in the article's Creative Commons licence and your intended use is not permitted by statutory regulation or exceeds the permitted use, you will need to obtain permission directly from the copyright holder. To view a copy of this licence, visit http://creativecommons.org/licenses/by/4.0/.

\section{References}

1. Adachi, T.: The classification of $\tau$-tilting modules over Nakayama algebras. J. Algebra 452, 227-262 (2016)

2. Adachi, T., Iyama, O., Reiten, I.: $\tau$-tilting theory. Compos. Math. 150(3), 415-452 (2014)

3. Araya, T.: Exceptional sequences over path algebras of type an and non-crossing spanning trees. Algebras Represent. Theory 16(1), 239-250 (2013)

4. Asai, S.: Semibricks. arXiv:1610.05860 (2016)

5. Assem, I., Skowronski, A., Simson, D.: Elements of the Representation Theory of Associative algebras: Volume 1: Techniques of Representation theory, vol. 65. Cambridge University Press, Cambridge (2006)

6. Bondal, A.I.: Representation of associative algebras and coherent sheaves. Izvestiya rossiiskoi akademii nauk. Seriya Matematicheskaya 53(1), 25-44 (1989) 
7. Buan, A.B., Marsh, R.J.: $\tau$-exceptional sequences. arXiv:1802.01169, pp. 24 (2018)

8. Bühler, T.: Exact categories. Expo. Math. 28(1), 1-69 (2010)

9. Corless, R.M., Gonnet, G.H., Hare, D.E.G., Jeffrey, D.J., Knuth, D.E.: On the Lambert W function. Adv. Comput. Math. 5(1), 329-359 (1996)

10. Crawley-Boevey, W.: Exceptional sequences of representations of quivers. Represent. Algebras (Ottawa, ON, 1992) 14, 117-124 (1993)

11. Gao, H., Schiffler, R.: On the number of support $\tau$,-tilting modules over Nakayama algebras. arXiv:2002.02990 (2020)

12. Garver, A., Igusa, K., Matherne, J.P., Ostroff, J.: Combinatorics of exceptional sequences in type A. arXiv: 1506.08927 (2015)

13. Gellert, L., Sanyal, R.: On degree sequences of undirected, directed, and bidirected graphs. Eur. J. Comb. 64, 113-124 (2017)

14. Gorodentsev, A.L., Rudakov, A.N., et al.: Exceptional vector bundles on projective spaces. Duke Math. J. 54(1), 115-130 (1987)

15. Gorodentsev, A.L.: Exceptional bundles on surfaces with a moving anticanonical class. Math. USSRIzvestiya 33(1), 67 (1989)

16. Igusa, K., Schiffler, R.: Exceptional sequences and clusters. J. Algebra 323(8), 2183-2202 (2010)

17. Igusa, K., Todorov, G.: Signed exceptional sequences and the cluster morphism category. arXiv:1706.02041 (2017)

18. Jasso, G.: Reduction of $\tau$-tilting modules and torsion pairs. Int. Math. Res. Not. 2015(16), 7190-7237 (2014)

19. Knuth, D.E., Pittel, B.: A recurrence related to trees. Proc. Amer. Math. Soc. 105(2), 335-349 (1989)

20. Komatsu, T., Ramírez, J.: Some determinants involving incomplete Fubini numbers. Analele Universitatii Ovidius Constanta-Seria Matematica 26(3), 143-170 (2018)

21. Mezo, I.: Periodicity of the last digits of some combinatorial sequences. J. Integer Seq. 17(2), 3 (2014)

22. Obaid, M.A.A., Nauman, S.K., Al Shammakh, W.S., Fakieh, W.M., Ringel, C.M.: The number of complete exceptional sequences for a Dynkin algebra. arXiv:1307.7573 (2013)

23. Polya, G., Read, R.C.: Combinatorial Enumeration of Groups, Graphs, and Chemical Compounds. Springer Science \& Business Media, Berlin (2012)

24. Pólya, G., et al.: Kombinatorische anzahlbestimmungen für gruppen, graphen und chemische verbindungen. Acta Math. 68, 145-254 (1937)

25. Ringel, C.M.: The braid group action on the set of exceptional sequences of a hereditary Artin algebra. Contemp. Math. 171, 339-339 (1994)

26. Riordan, J.: Combinatorial identities (1968)

27. Schiffler, R.: Quiver Representations. Springer, Berlin (2014)

28. Seidel, U.: Exceptional sequences for quivers of Dynkin type. Commun. Algebra 29(3), 1373-1386 (2001)

29. Sen, E.: Exceptional sequences and idempotent functions. Rocky Mountain J. Math. (2020)

30. Sen, E.: Weak exceptional sequences. Quaest. Math., 1-17 (2020)

31. Wilf, H.S.: Generatingfunctionology. AK Peters/CRC Press (2005)

Publisher's Note Springer Nature remains neutral with regard to jurisdictional claims in published maps and institutional affiliations. 